\title{
The relation between gas density and velocity power spectra in galaxy clusters: High-resolution hydrodynamic simulations and the role of conduction
}

\author{
M. Gaspari ${ }^{1}$, E. Churazov ${ }^{1,2}$, D. Nagai ${ }^{3,4}$, E. T. Lau ${ }^{3,4}$, and I. Zhuravleva ${ }^{5,6}$ \\ 1 Max Planck Institute for Astrophysics, Karl-Schwarzschild-Strasse 1, 85741 Garching, Germany \\ e-mail: mgaspari@mpa-garching.mpg.de \\ 2 Space Research Institute (IKI), Profsoyuznaya 84/32, 117997 Moscow, Russia \\ 3 Department of Physics, Yale University, New Haven CT 06520, USA \\ 4 Yale Center for Astronomy and Astrophysics, Yale University, New Haven CT 06520, USA \\ 5 Kavli Institute for Particle Astrophysics and Cosmology, Stanford University, 452 Lomita Mall, Stanford CA 94305-4085, USA \\ ${ }^{6}$ Department of Physics, Stanford University, 382 via Pueblo Mall, Stanford CA 94305-4060, USA
}

Received 21 April 2014 / Accepted 4 July 2014

\begin{abstract}
Exploring the power spectrum of fluctuations and velocities in the intracluster medium (ICM) can help us to probe the gas physics of galaxy clusters. Using high-resolution 3D plasma simulations, we study the statistics of the velocity field and its intimate relation with the ICM thermodynamic perturbations. The normalization of the ICM spectrum (related to density, entropy, or pressure fluctuations) is linearly tied to the level of large-scale motions, which excite both gravity and sound waves due to stratification. For a low 3D Mach number $M \sim 0.25$, gravity waves mainly drive entropy perturbations, which are traced by preferentially tangential turbulence. For $M>0.5$, sound waves start to significantly contribute and pass the leading role to compressive pressure fluctuations, which are associated with isotropic (or slightly radial) turbulence. Density and temperature fluctuations are then characterized by the dominant process: isobaric (low $M$ ), adiabatic (high $M$ ), or isothermal (strong conduction). Most clusters reside in the intermediate regime, showing a mixture of gravity and sound waves, hence drifting toward isotropic velocities. Remarkably, regardless of the regime, the variance of density perturbations is comparable to the $1 \mathrm{D}$ Mach number, $M_{1 \mathrm{D}} \sim \delta \rho / \rho$. This linear relation allows us to easily convert between gas motions and ICM perturbations $(\delta \rho / \rho<1)$, which can be exploited by the available Chandra, XMM data and by the forthcoming Astro-H mission. At intermediate and small scales (10-100 kpc), the turbulent velocities develop a tight Kolmogorov cascade. The thermodynamic perturbations (which can be generally described by log-normal distributions) act as effective tracers of the velocity field, in broad agreement with the Kolmogorov-Obukhov-Corrsin advection theory. The cluster radial gradients and compressive features induce a flattening in the cascade of the perturbations. Thermal conduction, on the other hand, acts to damp the thermodynamic fluctuations, washing out the filamentary structures and steepening the spectrum, while leaving the velocity cascade unaltered. The ratio of the velocity and density spectrum thus inverts the downtrend shown by the non-diffusive models, as it widens up to $\sim 5$. This new key diagnostic can robustly probe the presence of conductivity in the ICM. We produce X-ray images of the velocity field, showing how future missions (e.g. Astro-H, Athena) can detect velocity dispersions of a few $100 \mathrm{~km} \mathrm{~s}^{-1}(M>0.1$ in massive clusters), allowing us to calibrate the linear relation and to constrain relative perturbations down to just a few percent.
\end{abstract}

Key words. galaxies: clusters: intracluster medium - hydrodynamics - turbulence - conduction - methods: numerical -

X-rays: galaxies: clusters

\section{Introduction}

The power spectrum of perturbations and velocity in a given fluid has historically represented one of the crucial tools to understand and constrain the dominant astrophysical processes. In the cosmology field, the temperature fluctuations in the cosmic microwave background have allowed us to put precise constraints on the geometry and composition of the universe (e.g. through the acoustic spectral peaks; Planck Collaboration XVI 2014). Closer to our case, the observed electron density perturbations in the interstellar medium (ISM) have revealed a highly turbulent medium, showing a Kolmogorov power-law spectrum spanning more than 10 decades (Armstrong et al. 1981, 1995, and references therein). The observed power spectrum of the solar wind density, which is also consistent with the famous $-5 / 3$ slope, has further proven that turbulent processes are a key component shaping the dynamics of astrophysical plasmas (e.g. Woo \& Armstrong 1979; Marsch \& Tu 1990). In a similar way, the wealth of information contained in the power spectrum extracted from the hot plasma filling galaxy clusters can help us to significantly advance our knowledge of the intracluster medium (ICM) astrophysics.

In the context of galaxy clusters, Gaspari \& Churazov (2013; hereafter GC13) have shown for the first time that the power spectrum of the ICM density fluctuations linearly rises with the level of turbulent motions. Diffusive processes, as thermal conduction, instead fight to damp the cascade of perturbations. Many questions still remain to be tackled. In this work, we focus on the statistics and features of the velocity field in the stratified ICM, such as the power spectrum, the real-space and projected maps, and, in particular, its intimate relation with the thermodynamic perturbations. The ICM power spectrum can be viewed in various forms (e.g. Schuecker et al. 2004; Churazov et al. $2012)$ through the lenses of gas velocities $\left(\delta v / c_{\mathrm{s}}\right)$, density $(\delta \rho / \rho)$, 
entropy $(\delta K / K)$, or pressure $(\delta P / P)$ fluctuations, thus offering multiple joint constraints. Each physical process leaves marked imprints behind. The normalization of the perturbation spectrum is tied to the combined action of gravity and sound waves excited by large-scale (100s kpc) turbulence. At intermediate scales, the thermodynamic perturbations act as "tracers" of the eddy inertial cascade (in line with the classic advection theory by Obukhov 1949 and Corrsin 1951), while rising diffusivity conspire to decouple the tight relation. Faster gas motions alter the thermodynamic mode (from isobaric to adiabatic), changing the interplay between different fluctuations.

The linear relation between the density variance and the turbulent Mach number $M$ has been also observed in simulations of supersonic isothermal turbulence in homogeneous and periodic boxes (e.g. Padoan et al. 1997; Konstandin et al. 2012, and references therein), in connection with ISM studies. However, this relation purely arises from the high compression imparted by supersonic turbulence, creating shocks and sharp peaks (e.g. Kim \& Ryu 2005). In the subsonic regime, the compressibility drastically diminishes, and density perturbations fade as $M^{2}$ (Kowal et al. 2007). In the case of stratified galaxy clusters, our novel linear relation is instead tied to the radial gradients of entropy and pressure (Sect. 5), which is already developing in the subsonic regime - the realistic state of ICM turbulence $(M \sim 0.2-0.7$; e.g. Norman \& Bryan 1999; Lau et al. 2009; Vazza et al. 2009).

The velocity statistics of the diffuse medium is notoriously difficult to assess through observations. On the contrary, X-ray surface brightness images can robustly constrain the ICM density. Being able to convert between the spectra (or even just the normalization) of perturbations and velocity, is a powerful tool, which can be exploited by theoretical studies and by the large amount of available Chandra and XMM data. For instance, the quick estimate of the ICM turbulent velocities allows us to study the level of hydrostatic equilibrium in the hot halo (e.g. Vikhlinin et al. 2006), the transport and dilution of metals (e.g. Rebusco et al. 2005), the deposition of energy imparted by the active galactic nucleus (AGN) outflows (e.g. Churazov et al. 2004; Gaspari et al. 2011b, 2012b), the evolution of filaments and bubbles (e.g. Scannapieco \& Brüggen 2008), or the reacceleration of cosmic rays (e.g. Brunetti \& Lazarian 2007). On the other hand, being able to quickly assess the conductive state of the plasma allows us to constrain the survival of the cold/warm gas, which is crucial for star formation (e.g. McDonald \& Veilleux 2009) and black hole accretion (e.g. Gaspari et al. 2013), to study the quenching of cooling flows (e.g. Kim \& Fabbiano 2003) and the evolution of cosmic structures (e.g. Dolag et al. 2004).

We could soon take advantage of the inverse process, albeit more expensive. The upcoming Astro-H mission (Takahashi et al. 2010) and the future Athena (Nandra et al. 2013) will provide unprecedented detections of the turbulent velocity dispersion in the ICM (via line broadening) and bulk motions (via line shift; e.g. Inogamov \& Sunyaev 2003; Zhuravleva et al. 2012; Nagai et al. 2013; Tamura et al. 2014) down to Mach numbers $\sim 0.1$ for massive clusters (see Sect. 4). Reliable constraints on the gas motions allow us to accurately calibrate the above relation and to assess the level of density fluctuations, if the imaging is poor. For instance, apparently "relaxed" systems may host $\gtrsim 10$ percent density fluctuations, which may significantly alter the formation or regeneration of cool cores in clusters and bias the estimate of radial profiles to name a few interesting applications. The same spectral analysis can be extended to the gaseous halos of massive galaxies and groups.

The physics of the ICM is a strongly debated topic. Turbulence has been mainly studied by means of cosmological and isolated simulations (e.g. Norman \& Bryan 1999; Dolag et al. 2005; Kim \& Ryu 2005; Nagai et al. 2007; Lau et al. 2009; Vazza et al. 2009; Valdarnini 2011; Borgani \& Kravtsov 2011; Miniati 2014; Schmidt et al. 2014; Shi \& Komatsu 2014). Similarly, diffusion processes as conduction have been mainly investigated via theoretical studies (Chandran \& Cowley 1998; Narayan \& Medvedev 2001; Zakamska \& Narayan 2003; Ruszkowski \& Oh 2010, 2011; Voigt \& Fabian 2004; Roediger et al. 2013; Smith et al. 2013; ZuHone et al. 2013). Instead, observations have a hard time in resolving and constraining such processes through local features, granting in the last decade only a few estimates (Ettori \& Fabian 2000; Markevitch \& Vikhlinin 2007; Forman et al. 2007; Eckert et al., in prep.). However, we are now able to retrieve the statistics of density/pressure fluctuations in the ICM (Schuecker et al. 2004; Churazov et al. 2012; Sanders \& Fabian 2012), which allows us to probe the gas physics without the need to resolve local structures.

In Churazov et al. (2012), we outlined possible effects that can contribute to density fluctuations: turbulence (via the Bernoulli term $\propto M^{2}$ or via sound waves), entropy variations (due to mergers or turbulence), perturbations of the gravitational potential, metallicity variations, and AGN bubbles. Using 3D high-resolution plasma simulations, we focus in this work on the role of turbulence and thermal diffusivity and the driven thermodynamic perturbations, including entropy and pressure variations. Controlled experiments allow us to discriminate the exact contribution of each included physics. In a companion paper (Zhuravleva et al. 2014, hereafter Z14), we analyze the density perturbations in cosmological AMR simulations and we focus on the role of gravity waves. At the price of lower resolution, we are thus able to include the turbulence driving led by mergers (Sect. 5.3).

This work is structured as follows. In Sect. 2, we review the main physical and numerical ingredients of the simulated models. In Sect. 3, we present the power spectrum of velocities and density fluctuations by focusing on their tight connection and key features (normalization, cascade, and damping). In Sect. 4, we analyze the real-space properties of the velocity and perturbation field, showing what X-ray observations are able to detect. In Sect. 5, we throughly discuss the physical interpretation of the power spectrum, the interplay of $g$-waves and $p$-waves, along with the development of the spectral cascade of all thermodynamic perturbations, in relation to the advection theory of tracers. In Sect. 6, we summarize the results and remark how the ICM power spectrum can be exploited by future observations and theoretical studies to probe the physics of the diffuse medium with high precision.

\section{Physics and numerics}

The implemented physics and numerics are described in depth in Gaspari \& Churazov (2013; Sect. 2), to which the reader is referred for the complete details. Here we summarize the essential features.

The initial conditions for the hot gas are modeled following the latest XMM observed temperature and density radial profiles of Coma cluster $\left(\beta\right.$-model with core radius $r_{\mathrm{c}}=272 \mathrm{kpc}$ and index 0.75 ). Given the high ICM temperature, $T \sim 8.5 \mathrm{keV}$, and low electron number density, $n_{\mathrm{e}} \sim 4 \times 10^{-3}$, Coma serves as an excellent laboratory to study the effects of conduction and turbulence without being strongly influenced by radiative cooling or AGN feedback. The hot gas is initialized in hydrostatic equilibrium by providing a gravitational potential appropriate for a massive cluster in the $\Lambda \mathrm{CDM}$ universe with a virial mass 
$M_{\text {vir }} \sim 10^{15} M_{\odot}\left(r_{500} \sim 1.4 \mathrm{Mpc}\right.$, which is covered by the width of the $3 \mathrm{D}$ box).

The conservative hydrodynamics equations are integrated with the Eulerian code FLASH4 (Fryxell et al. 2000) by using a third order scheme (piecewise parabolic method) in the framework of the unsplit flux formulation. The ICM plasma has an adiabatic index $\gamma=5 / 3$ and mean atomic weight $\mu \simeq 0.62$. We chose the numerically expensive uniform grid $\left(512^{3}\right)$, instead of adaptive cells, to remove any substantial, spurious diffusivity due to the refinement/derefinement. The resolution is $\Delta x \simeq 2.6 \mathrm{kpc}$, which is roughly on the scale of the (unmagnetized) plasma mean free path, and mimics a slightly suppressed Spitzer viscosity. Boundary zones have a Dirichlet condition, which are fixed by the large-scale radial profile; inflow is prohibited. In addition to hydrodynamics, we add turbulence driving, thermal conduction, and electron-ion equilibration as source terms.

Injection of subsonic, solenoidal turbulence is modeled with a spectral forcing scheme that generates a statistically stationary velocity field (GC13, Sect. 2.2), which is based on an OrnsteinUhlenbeck random process. The amplitudes of the driven acceleration are evolved in Fourier space and then directly converted to physical space. Since observations (Schuecker et al. 2004; Churazov et al. 2008; de Plaa et al. 2012; Sanders \& Fabian 2013) and simulations (Norman \& Bryan 1999; Lau et al. 2009; Vazza et al. 2009, 2011; Gaspari et al. 2012b; Schmidt et al. 2014; Shi \& Komatsu 2014) show that the ICM turbulent energies are $\sim 3-30$ percent of the thermal energy, we test subsonic Mach numbers in the range of $M \equiv \sigma_{v} / c_{\mathrm{s}} \sim 0.25-0.75$, where $\sigma_{v}$ is the $3 \mathrm{D}^{1}$ velocity dispersion (average sound speed of Coma is $c_{\mathrm{s}} \simeq 1500 \mathrm{~km} \mathrm{~s}^{-1}$ ). The source of turbulence can be various, which includes cosmological flows/mergers, galaxy motions, and feedback processes. The former usually dominate by affecting large volumes beyond the core (e.g. Shi \& Komatsu 2014; Z14) and they are typically related to a solenoidal flow (e.g. Miniati 2014). We thus stir the gas on large scales with a typical injection peak $L \sim 600 \mathrm{kpc}$ (in a few runs $\sim 300 \mathrm{kpc}$ ), and we let turbulence to naturally cascade. The turbulence timescale as a function of physical scale $l$ is the eddy turnover time $t_{\text {turb }} \simeq\left(L^{1 / 3} / \sigma_{v, L}\right) l^{2 / 3}$, using the Kolmogorov scaling $\sigma_{v} \propto l^{1 / 3}$. Since turbulence is kept subsonic, dissipational heating is subdominant $t_{\text {diss,heat }} \sim M^{-2} t_{\text {turb }}$ (e.g. Ruszkowski \& Oh 2011) on timescales on order of the eddy turnover time. We recall that turbulence acts as an effective diffusivity on entropy with a coefficient $D_{\text {turb }} \sim \sigma_{v} l$ (e.g. Dennis \& Chandran 2005).

The conduction of thermal energy, due to the plasma electrons, is driven by a flux $\boldsymbol{F}_{\text {cond }}=-f \kappa_{\mathrm{S}} \boldsymbol{\nabla} T_{\mathrm{e}}$ with a conductivity $\kappa_{\mathrm{S}} \simeq 5 \times 10^{-7} T_{\mathrm{e}}^{5 / 2} \mathrm{erg} \mathrm{s}^{-1} \mathrm{~K}^{-1} \mathrm{~cm}^{-1}$ (Spitzer 1962). The related diffusivity and timescale is $D_{\text {cond }}=f \kappa_{\mathrm{S}} / 1.5 n_{\mathrm{e}} k_{\mathrm{B}}$ and $t_{\text {cond }}=l^{2} / D_{\text {cond }}$, respectively. The conductive flux saturates as $F_{\text {sat }} \propto n_{\mathrm{e}} T_{\mathrm{e}}^{3 / 2}$, whenever the temperature scale height is smaller than the electron mean free path. We use an advanced implicit solver, which allows for long, Gyr integration times. The magnetohydrodynamic (MHD) simulations (e.g. Ruszkowski \& Oh 2010) show that the outcome of subsonic turbulence is a tangled magnetic field with small kpc coherence length (see also constraints in Kim et al. 1990). On scales larger than the coherence length, the average suppression due to anisotropic conduction and magnetic microinstabilities can be parametrized with the so-called $f$ factor, commonly known as $f \sim 10^{-3}-10^{-1}$ (GC13, Sect. 2.1.1). Using the effective isotropic conductivity has the advantage of modeling any level of suppression affecting

\footnotetext{
The volume-weighted or mass-weighted 3D Mach number is very similar within $\lesssim 3$ percent accuracy.
}

the bulk of the ICM. The MHD runs only provide the geometric suppression above the plasma mean free path $\lambda$; in a chaotic atmosphere, it is typically $f \sim 1 / 3$ (Ruszkowski \& Oh 2010; see also Narayan \& Medvedev 2001). However, line wandering, magnetic mirrors, and other plasma microinstabilities, which are well below $\lambda$ can strongly suppress the transport of heat down to $f \sim 10^{-3}$ (Rechester \& Rosenbluth 1978; Chandran \& Cowley 1998; Komarov et al. 2014). The survival of cold fronts, bubbles, and cold gas (Sect. 1), in conjunction with our GC13 spectral analysis, point toward strongly suppressed values, $f \sim 10^{-3}$.

We integrate both the electron and ion temperature equation, since equilibration times can become considerable in a hot plasma ( $\left.t_{\mathrm{ei}} \gtrsim 50 \mathrm{Myr}\right)$. The heat exchange rate is $\propto\left(T_{\mathrm{e}}-T_{\mathrm{i}}\right) / t_{\mathrm{ei}}$, using a Spitzer equilibration time $t_{\mathrm{ei}} \propto T_{\mathrm{e}}^{3 / 2} / n_{\mathrm{e}}$ (cf. GC13). The $2 \mathrm{~T}$ modeling allows us to prevent the formation of spurious perturbations due to the unphysical instantaneous transfer of heat.

\section{The ICM power spectrum: velocity and $\delta \rho / \rho$}

We now describe the results of the simulated models, which focus on the spectral and real-space properties of the turbulent velocity, in relation with the statistics of gas density perturbations, $\delta \rho / \rho$. Gas density is indeed the primary astrophysical observable, which is directly extracted from the X-ray surface brightness. As thoroughly discussed in Sect. 5, the perturbations would be actually more evident through entropy (for low $M$ ) or pressure (for high $M$ ), and then by retrieving $\delta \rho / \rho$ via the main thermodynamic mode (isobaric, isothermal, or adiabatic). Unfortunately, $K$ and $P$ are difficult X-ray observables to constrain. Nevertheless, the spectrum of the leading "tracer" is tied to velocities in a very similar manner, although the underlying cause differs, granting a fairly universal $M-\delta \rho / \rho$ relation (Sects. 5.1.1-5.1.2).

We first retrieve the characteristic amplitude of total velocity normalized to $c_{\mathrm{s}} \simeq 1500 \mathrm{~km} \mathrm{~s}^{-1}$. It is convenient to use the characteristic amplitude, instead of the power spectrum $P(k)$ or energy spectrum $E(k)$, since its units are the same of the variable in real space. The amplitude spectrum is defined as

$A(k) \equiv \sqrt{P(k) 4 \pi k^{3}} \equiv \sqrt{E(k) k}$,

where $k=\sqrt{k_{x}^{2}+k_{y}^{2}+k_{z}^{2}} \equiv l^{-1}\left(\mathrm{kpc}^{-1}\right)$. No major bulk motion is present in our box (average velocity $\sim 0$ ); the velocity dispersion is strictly associated with the turbulence driving. The relative perturbations are divided by the underlying background radial profile, for example $\delta \rho / \rho=\rho / \rho_{\mathrm{b}}-1$ (GC13, Sect. 2.7). Except for mild deviations (Sect. 5.1.3), the turbulence field can be considered isotropic as a first order approximation, allowing us to use the conversion $v_{1 \mathrm{D}} \sim v / \sqrt{3}$. All the power spectra are computed with "Mexican Hat" filtering (Arévalo et al. 2012) instead of performing Fourier transforms (GC13, Appendix A for a comparison), which can lead to spurious features due to the box non-periodicity.

In Fig. 1, we show the retrieved characteristic amplitude of $v / c_{\mathrm{S}}$ (blue; we notice that $A_{v}=\sqrt{A_{v_{\mathrm{x}}}^{2}+A_{v_{\mathrm{y}}}^{2}+A_{v_{\mathrm{z}}}^{2}}$ ), which is superposed to the amplitude of density perturbations (red), after reaching a statistical steady state $\left(\gtrsim 2 t_{\text {turb }}\right)$. From the top left panel, the models have increasing turbulence: weak $(M \sim 0.25)$, mild $(M \sim 0.5)$, and strong $(M \sim 0.75)$. The ratio of turbulent to thermal energy is $3.5,14$, and 31 percent $\left(E_{\text {turb }} \simeq 0.56 M^{2} E_{\text {th }}\right)$, respectively. The last model (bottom right panel) tests weak turbulence with half the reference injection scale $(\sim 300 \mathrm{kpc})$. The 

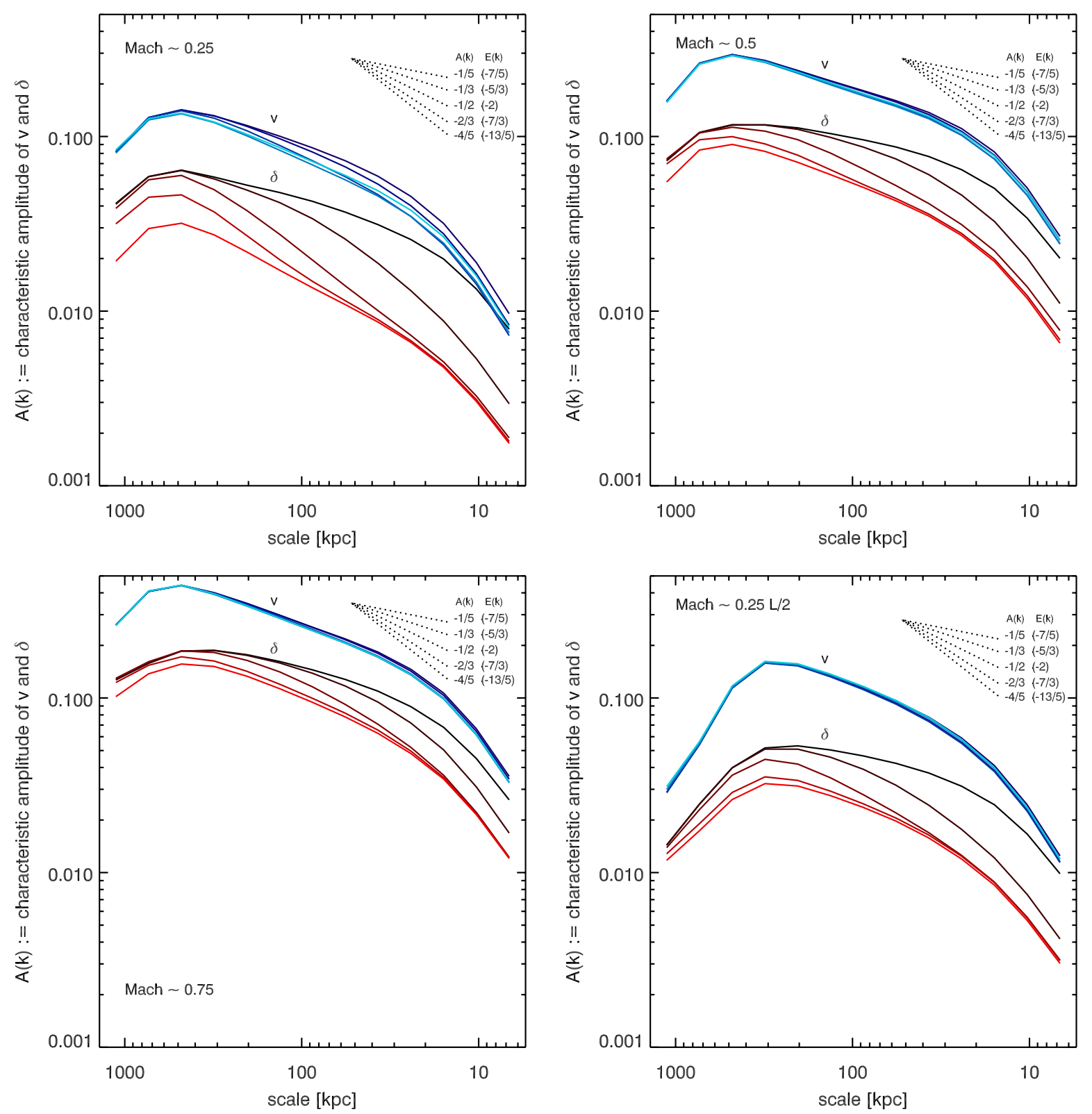

Fig. 1. Characteristic amplitude $\left(A(k)=\sqrt{P(k) 4 \pi k^{3}}\right)$ of $\delta \rho / \rho$ (red) and $v / c_{\mathrm{s}}$ (blue) after reaching a statistical steady state $\left(\sim 2 t_{\text {turb }}\right)$ with the same level of continuous stirring. From top left: models with weak $(M \sim 0.25)$, mild $(M \sim 0.5)$, and strong $(M \sim 0.75)$ turbulence; the last model (bottom right $)$ has half the reference injection scale $(\sim 600 / 2 \mathrm{kpc})$ using $M \sim 0.25$. From dark to bright line color, the level of conduction increases by a factor of 10: $f=0$ (hydro), $10^{-3}, 10^{-2}, 10^{-1}, 1$. The evolution is overall self-similar by varying the strength of turbulence or the injection scale. Density perturbations are an effective tracer of the velocity field, especially on large scales, with normalization $A_{v_{1}} \approx 1.3 A_{\rho}$ (at $L \sim 600 \mathrm{kpc}$ ). On smaller scales, $\delta \rho / \rho$ displays a cascade shallower than the Kolmogorov slope followed by velocities. Remarkably, conduction strongly damps density perturbations but leaves the velocity cascade unaltered, thus inverting the $A_{v}(k) / A_{\rho}(k)$ ratio (Fig. 2).

overall behavior of the spectra related to density, velocities, and their ratio (Fig. 2) is fairly self-similar over different Mach numbers and injection scales. We covered the details of the $\delta \rho / \rho$ spectrum in GC13. We focus here on the turbulent velocity and their relationship.

The normalization of the velocity spectrum sets the level of perturbations, which is tied to the $A(k)$ peak. In Fig. 2, we better illustrate the $A_{v}(k) / A_{\rho}(k)$ ratios. The hydro $f=0$ runs (top) show a converging maximum value $A_{v} / A_{\rho} \approx 2.3$ (using the $1 \mathrm{D}$ velocity $A_{v_{1 \mathrm{D}}} / A_{\rho} \approx 1.3$ ), implying that a stronger turbulence linearly induces larger density fluctuations. This is a key result that allows us to quickly estimate ICM perturbations via the leading turbulent motions and vice versa. For low $M$, gravity waves mainly produce entropy perturbations, as $\delta K / K \propto v_{1 \mathrm{D}}$ (see Sect. 5.1.1). For slow motions, the isobaric mode is respected, hence $v_{1 \mathrm{D}} \propto \gamma \delta \rho / \rho$, as also simulated. For $M \gtrsim 0.5$ (see Sect. 5.1.2), compressive sound waves start to significantly contribute: entropy perturbations remain constant, while $\delta P / P$ increases and sustains the same linear relation, albeit the thermodynamic mode smoothly shifts toward the adiabatic regime. In Sect. 5, we thoroughly discuss all the thermodynamic perturbations (Figs. 8, 9) and the underlying physical interpretation.

For applications in other studies, it is important to note that, proper attention should be paid to the conversion to adopt, given 
M. Gaspari et al.: The ICM power spectrum - gas perturbations trace velocities while decouple via conduction
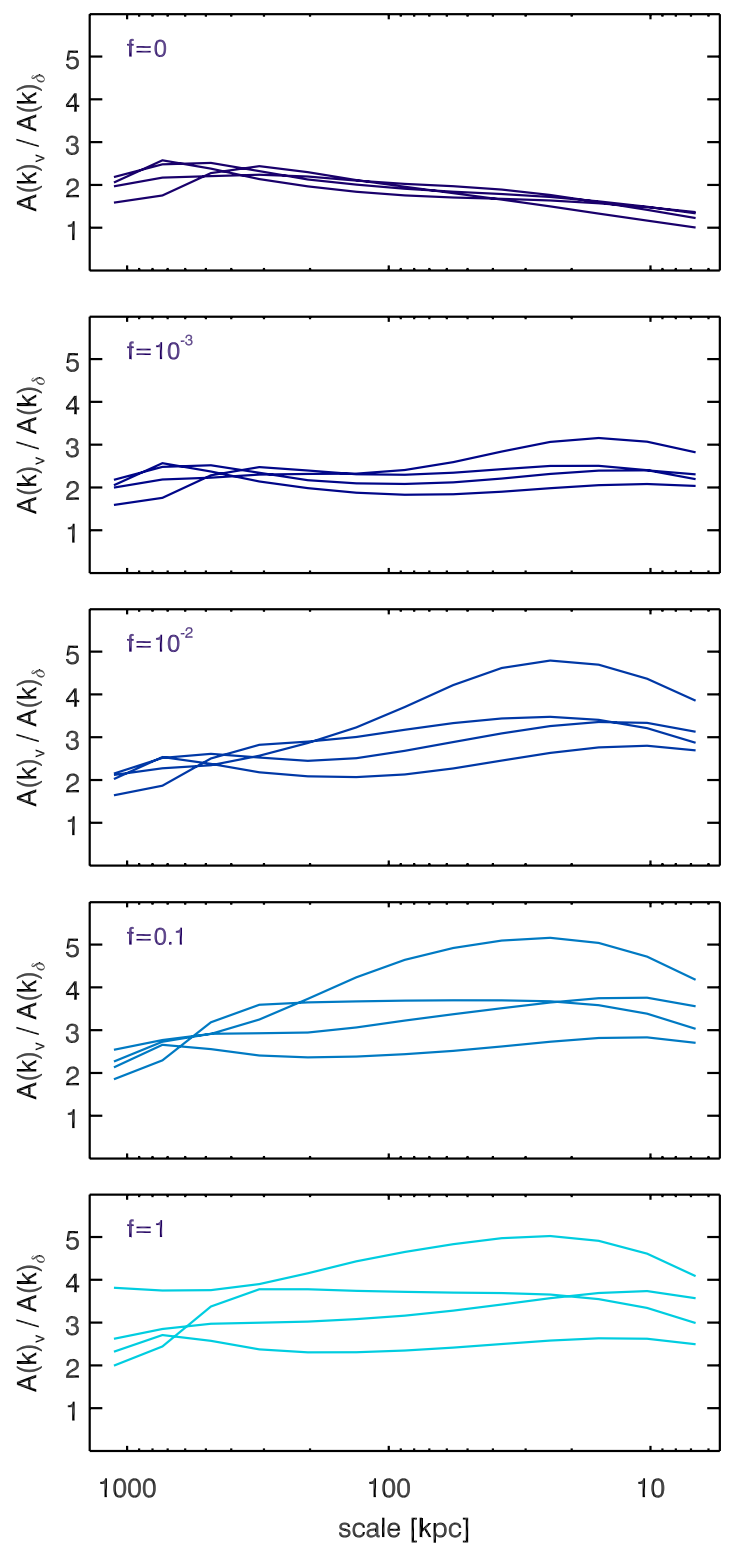

Fig. 2. Ratio of the power spectrum related to total velocity and density perturbations for all the computed models. Each panel groups the models with identical conductivity but different $M$ (same colors as in Fig. 1). The $A_{\rho}$ for $L / 2$ runs is rescaled by a factor $2^{1 / 3}$ to emphasize slope similarities. In the hydro runs, the ratio is very tight, decreasing from $\sim 2.5$ to roughly unity, near the dissipation scale. An increasing ratio instead marks the presence of significant conduction. Lower Prandtl numbers $\left(P_{\mathrm{t}} \propto M / f\right)$ lead to wider scatter with ratios up to $\sim 5$. The $A_{v} / A_{\rho}$ ratio is a new key diagnostics able to unveil the presence of substantial conductivity in the ICM.

the initial quantity or observable. Shifting from the spectral to physical integrated ${ }^{2}$ quantities, the velocity/density ratio remains the same. However, if we relate the real-space Mach number (i.e. the total variance) to the spectral peak, as in GC13, the conversion to use is $M \approx 4 A(k)_{\rho, \max }$ (assuming $L \sim 600 \mathrm{kpc}$ ). Furthermore, turbulence cannot create relative density perturbations with an amplitude higher than roughly the 1D Mach number (Sect. 5). If significantly violated, this would indicate that the perturbation or velocity field has been contaminated by the

\footnotetext{
2 The total variance can be computed by integrating $P(k) 4 \pi k^{2} \mathrm{~d} k$ over the whole range of scales. The total variance (as the $M$ value) is typically $\sim 1.7$ times higher than the $A(k)$ peak here.
}

unfiltered background profile or laminar flows (which are particularly complex in unrelaxed systems). Similarly, strong inhomogeneities must be properly removed: the linear relation applies to relatively small perturbations $\delta \rho / \rho<1$ and not to features as cold fronts or buoyant bubbles.

Below the injection scale, $A_{v}$ and $A_{\rho}$ continue to be tightly related in the non-diffusive models (Fig. 2). The ratio is independent of $M$, as indicated by the tight scatter. It is remarkable that the density acts as an effective "tracer" of the velocity field, developing a similar inertial cascade. This is also true for the leading entropy/pressure perturbations. The phenomenon can be explained via the classic theory of advection of passive tracers in turbulent media, $A(k)_{\rho} \propto A(k)_{v}$ (Obukhov 1949; Corrsin 1951; see Sect. 5.2). On the other hand, in the hydro runs we observe that the ratio steadily declines as $l^{0.13}$, which reaches about unity near the dissipation scale. The decrease is associated with a shallower cascade of perturbations due to the initial radial gradients, compressive features, and differences in the diffusivity of the "tracer" (Sect. 5.2.1). In Fig. 3 (top), we show a test with $2 \times$ lower resolution (i.e. $2 \times$ higher effective viscosity). Besides the good large-scale convergence, the run clarifies that the density/tracer is susceptible to diffusivity in a slightly different way compared with $v$; hence, we expect departures from the classic tracers theory. We notice also how the cascade of density perturbations is not a perfect power law but tends to exponentially decline, even in the hydro run.

Using half the reference injection scale, the $A_{v}$ peak is analogous to the that of the reference run (conserving $M \sim 0.25$ ), while the density perturbations slightly decrease by $\sim 2^{1 / 3}$, that is the previous cascade truncated at $L / 2$. Stirring smaller scales reduces the influence of gravity waves, since the zone where the turbulence frequency $\left(\propto L^{-1 / 3}\right)$ is shorter than the buoyancy frequency shrinks (Fig. 7). By varying the injection scale between $L^{\prime}=L \equiv 600 \mathrm{kpc}, L^{\prime}=L / 2$, and $L^{\prime}=L / 3$ we find that correcting the ratio by a factor $\sim\left(L^{\prime} / L\right)^{1 / 3}$ restores the normalization to a universal value (Fig. 2, top). The development of the shorter cascade is also hindered by the progressive proximity to the dissipation scale.

A key result is the substantial decoupling of velocities and density perturbations, as we increase the level of conduction (dark to bright line color: $f=0,10^{-3}, 10^{-2}, 10^{-1}$, 1; Figs. 1, 2). In other words, the quick transfer of heat damps density fluctuations (the forming overdensities quickly re-expand due to the temperature increase), while it leaves the turbulent velocity cascade or momentum transfer unaltered. The rising $A_{v} / A_{\rho}$ ratio (Fig. 2, top to bottom) is a crucial result that provides a new constraint on the conductive state of the ICM, in addition to the slope of the spectrum. It also breaks any minor degeneracy that strong conduction $(f \gtrsim 0.1)$ may induce in the spectrum, due to the damping of power at large scales (slightly flattening the cascade). The upcoming Astro-H mission should provide important constraints on the ICM turbulent velocities; combined with high-quality determinations of density perturbations via Chandra/XMM (and Athena), our knowledge of the ICM physics could significantly improve, exploiting the $A_{v} / A_{\rho}$ diagnostic.

The velocity cascade follows the Kolmogorov index $(A(k) \propto$ $k^{-1 / 3}$ or $\left.E(k) \propto k^{-5 / 3}\right)$ in all runs, except with weak turbulence, where it becomes slightly steeper, though with increased scatter. Considering the substantial stratification, it is remarkable that classic Kolmogorov theory consistently applies to a cluster atmosphere (see also Vazza et al. 2011; Valdarnini 2011). The density spectrum instead displays a steep decay toward $A_{\rho} \propto k^{-1 / 2}$, as the turbulent Prandtl number $P_{\mathrm{t}} \equiv t_{\text {cond }} / t_{\text {turb }} \lesssim 100$ (see 

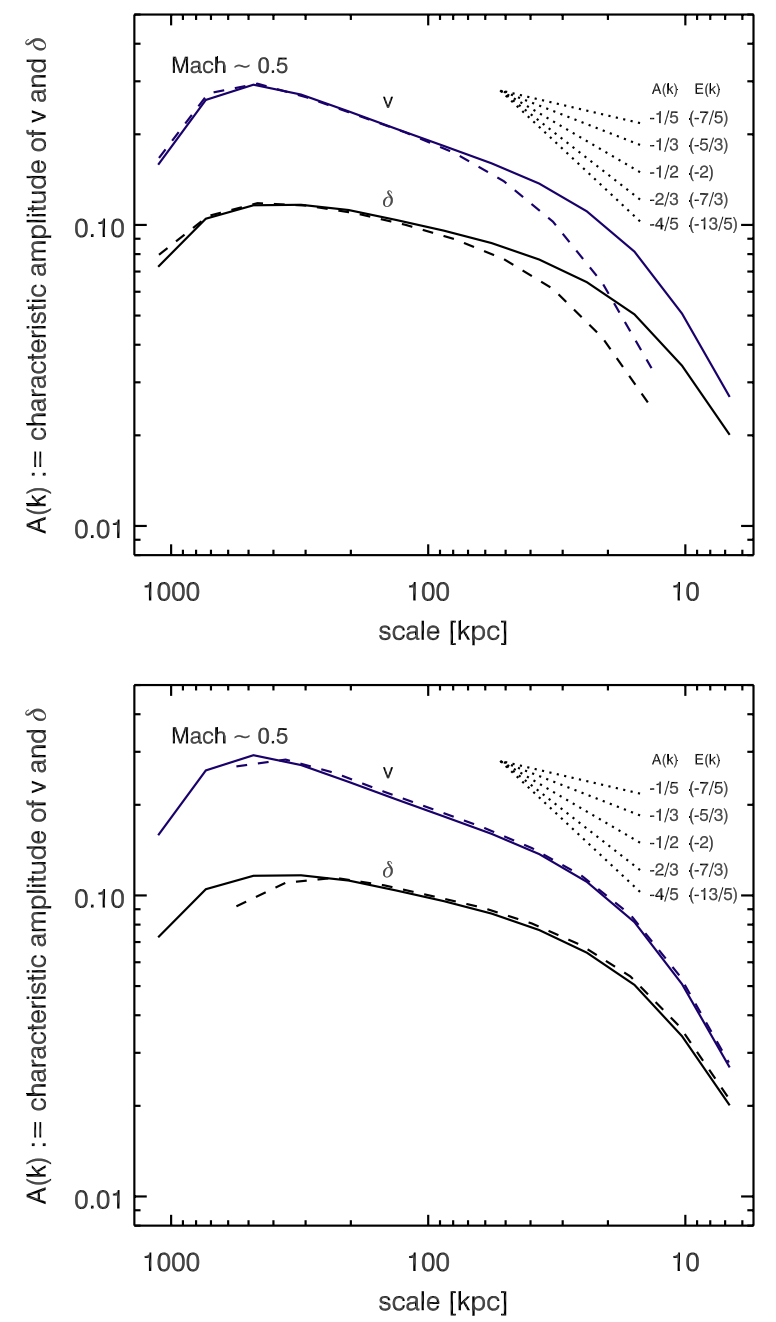

Fig. 3. Top: characteristic amplitude of $v / c_{\mathrm{s}}$ and $\delta \rho / \rho$ for the hydro model with $M \sim 0.5$, doubling the numerical viscosity, i.e. using $2 \times$ lower resolution (similar to the Spitzer value; dashed lines). Spectra are convergent, except at small scales where the increased numerical diffusivity damps both $v$ and $\delta \rho / \rho$ at about twice the original dissipation scale. The density cascade is affected in a slightly different way by the larger diffusivity; some deviations from the classic advection theory of tracers are thus expected (Sect. 5.2.1). Bottom: spectrum of the above model extracted in the full box (solid) and in the center (dashed; $\left.<r_{500} / 4\right)$, where stratification is less prominent. Turbulence and density perturbations are overall homogeneous, despite the cluster stratification (compare with Fig. 4).

GC13 $\left.{ }^{3}\right)$. Such a steepening induces $A_{v} / A_{\rho}$ to become gradually shallower ${ }^{4}$, inverting the trend for $f \gtrsim 10^{-2}$ (Fig. 2, third panel). This can be explained in terms of the advection theory of tracers (Sect. 5.2), where the diffusivity of the scalar has no effect on the velocity cascade. The scatter of $A_{v} / A_{\rho}$ rises with decreasing Prandtl number, that is with stronger conduction and weaker turbulence $\left(P_{\mathrm{t}} \propto M / f\right)$. Conductivity with $f \gtrsim 0.1$ can indeed stifle the regeneration of perturbations by a factor of 2-4 over a large range of scales. Figure 2 confirms that any substantial

\footnotetext{
3 For the weak turbulence run with $f=1, P_{\mathrm{t}} \sim 1$ at $L=600 \mathrm{kpc}$. We note $P_{\mathrm{t}}$ can be also seen as a turbulent Peclet number, if turbulence is interpreted as an advection rather than a diffusion process.

4 In general, significant diffusivity (especially numerical) acting on both $\rho$ and $v$ tends to align the two spectra even on small scales, which is a common feature we found in cosmological simulations (Z14 and Fig. 3).
}

conductivity in the ICM clearly emerges in the $A_{v} / A_{\rho}$ diagnostic, showing values up to $\approx 5$ and 3 for weak and strong turbulence, respectively, even at scales of $100 \mathrm{spc}$.

\section{Real-space properties and X-ray constraints}

Before delving into the theoretical interpretation (Sect. 5), it is worth understanding the real-space properties related to turbulence and perturbations of density (or the "tracer"), and what X-ray observations can detect through the spectral line broadening and the projected images. As reference, we consider the models with $M \sim 0.5\left(E_{\mathrm{turb}} / E_{\mathrm{th}} \sim 0.14\right.$, a common cluster regime; e.g. Schuecker et al. 2004; Lau et al. 2009; GC13 Sect. 4.3).

In Fig. 4, we compare the mid-plane cross-sections of $\delta \rho / \rho$ (left) and magnitude of total velocity (middle). The key result is the progressive smoothing of density fluctuations raising the level of conduction, while the turbulent velocity field remains unaltered (top to bottom panels: $f=0,10^{-3}, 10^{-2}, 10^{-1}$ ). In the hydro run, the perturbation field shows a complex morphology of filamentary structures, which are produced by the turbulent velocity field and later deformed by Kelvin-Helmholtz and Rayleigh-Taylor instabilities. The rolls and filaments are almost washed out in the presence of strong conduction $(f \gtrsim$ 0.1 ), transforming the perturbations from isobaric to isothermal (Sect. 5.1.1). On the other hand, strong conductivity cannot completely wipe out fluctuations (bottom maps). While entropy fluctuations decrease, compressive pressure perturbations still maintain the same level (Fig. 9, bottom). In other words, strong conduction can also promote minor fluctuations, due to the fast transfer of heat and change of compressibility in the medium.

All the velocity maps (middle) are remarkably similar, both statistically and locally, with minute differences only if $f \gtrsim 0.1$. As density fluctuations, the turbulent velocities do not show any major difference within or outside the cluster core, signaling a significant level of homogeneity. To be more quantitative, we extracted the spectra only from the cluster center $\left(r<r_{500} / 4\right)$, where stratification is less prominent. As shown in Fig. 3 (bottom), both the $\delta \rho / \rho$ and velocity spectra are similar to those computed in the full box. At the largest scale, $\delta \rho / \rho$ experiences a minor decline $(\sim 10 \%)$ partly because the entropy/pressure profile is shallower in the core and partly due to the limited statistics related to the smallest modes. Concerning isotropy, the realspace maps also do not highlight major deviations. However, transforming the velocity field in spherical coordinates, we retrieve mild anisotropies (Fig. 10). As $M<0.5$, the large-scale velocities become slightly more tangential, due to the stronger influence of stratification (Sect. 5.1.3). Instead, stronger turbulence (and conduction) increases the relevance of sound waves, restoring isotropy.

Figure 4 points out that the phases of the perturbation field are not coincident with that of the velocity field, although the two fields are strongly correlated in amplitude. The Pearson coefficient indicates a negligible anticorrelation between $\delta \rho / \rho$ and total velocity $(R<-0.2)$, in all models. In other words, the density filaments are not strictly tied to a local high velocity. This is expected, since the cause of fluctuations is the turbulence driving, while $\delta \rho / \rho$ plays the role of the tracer in a continuously chaotic environment.

We analyzed the volumetric PDF of the logarithmic density fluctuations, $\ln (1+\delta \rho / \rho)$. The thermodynamic fluctuations can be generally described by a log-normal distribution (see example in Fig. 5) with small corrections due to high-order moments 
M. Gaspari et al.: The ICM power spectrum - gas perturbations trace velocities while decouple via conduction
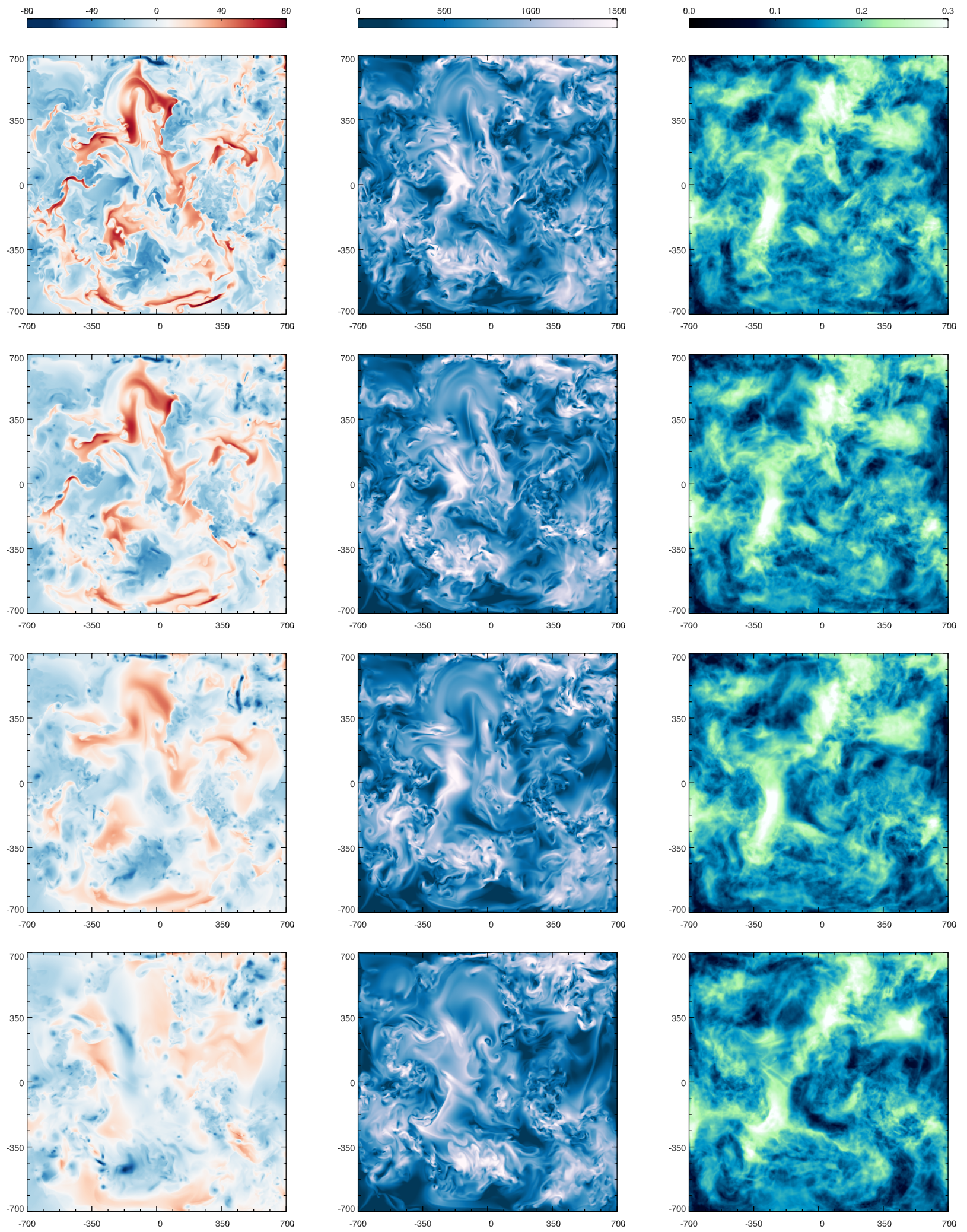

Fig. 4. Left: mid-plane cross-sections of $\delta \rho / \rho$ (percent) for the models with $M \sim 0.5$. From top to bottom: increasing conduction with $f=$ 0 (hydro), $10^{-3}, 10^{-2}, 10^{-1}$ (the latter very similar to the $f=1$ run). Middle: same cross-sections but for the module of total velocity (km s ${ }^{-1}$ ). The hydro runs show sharp filamentary density structures produced by the turbulent velocity field, which are later deformed by Kelvin-Helmholtz and Rayleigh-Taylor instabilities. Strong conduction damps these perturbations, while leaving the Kolmogorov cascade of turbulent eddies unaltered. The $v$ and $\delta \rho / \rho$ fields have correlated amplitude but different phases (the density field is the tracer). Right: observed relative line broadening (percent) due to turbulent motions along the $y$-axis view, $\Delta E / E_{0} \equiv \sqrt{2} \sigma_{1 \mathrm{D} \text {,ew }} / c$, where $\sigma_{1 \mathrm{D} \text {,ew }}$ is the projected X-ray emission-weighted velocity dispersion. The forthcoming Astro-H telescope should be able to detect projected turbulent velocities above $\sim 200 \mathrm{~km} \mathrm{~s}^{-1}$ or $\Delta E / E_{0}>0.1$ percent $(F W H M=1.66 \Delta E)$, using the Fe XXV line. 


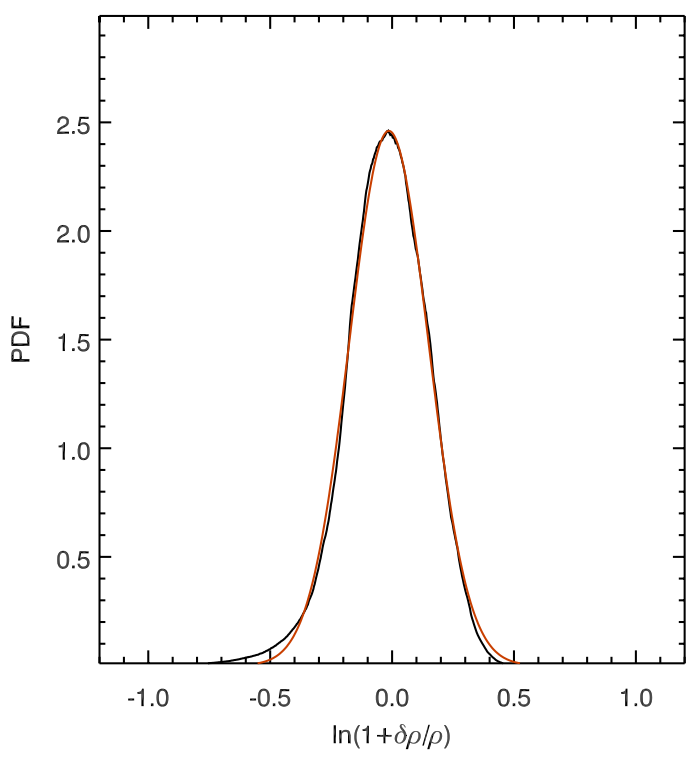

Fig. 5. Volumetric PDF of the logarithmic density fluctuations, $\ln (1+$ $\delta \rho / \rho$ ), for the run with $M \sim 0.5$ and $f=0.1$ (black). The thermodynamic fluctuations can be generally described by a log-normal distribution (red line) with small corrections due to high-order moments (skewness and kurtosis).

(skewness and kurtosis), thus strengthening the role of the power spectrum. The log-normal distribution and weak non-Gaussian contributions are consistent with classic turbulence studies that test solenoidal stirring, albeit in non-stratified and controlled boxes (Federrath et al. 2010, and references therein), and with ICM studies (Kawahara et al. 2007; Zhuravleva et al. 2013). Significant deviations start to arise with highly compressive turbulence, mainly affecting the wings (e.g. Kowal et al. 2007). We defer the study of high-order moments to future work.

What can be inferred from X-ray observations? Besides X-ray imaging (see surface brightness maps in GC13, Fig. 4, or Churazov et al. 2012), X-ray energy spectra can provide crucial constraints on turbulence, in particular by considering the forthcoming Astro-H mission (e.g. Inogamov \& Sunyaev 2003; Zhuravleva et al. 2012; Tamura et al. 2014). We have two important tools to exploit: one is the broadening of the spectral line, and the other is the line shift. In Fig. 4 (right), we show the observed line broadening due to turbulent motions along the $y$-axis view. The Doppler broadening relative to the line rest energy, $E_{0}$, is defined ${ }^{5}$ as $\Delta E / E_{0} \equiv \sqrt{2} \sigma_{1 \mathrm{D} \text {,ew }} / c$. The observed $1 \mathrm{D}$ velocity dispersion is computed as $\sigma_{1 \mathrm{D}, \text { ew }}^{2}=E\left[v_{1 \mathrm{D}}^{2}\right]-E^{2}\left[v_{1 \mathrm{D}}\right]$, where $E[x]$ is the X-ray emission-weighted average along the line of sight, using emissivity $n_{\mathrm{e}} n_{\mathrm{i}} \Lambda(T)$ with X-ray threshold $T_{\mathrm{x}} \gtrsim 0.3 \mathrm{keV}$ (Gaspari et al. 201a). The related full width of the line at half maximum is $F W H M \equiv 2 \sqrt{\ln 2} \Delta E \simeq 1.66 \Delta E$. The projected maps appear partly different from the cross-sections, but the statistics, like the velocity dispersion, is the same after deprojection. Astro-H will be able to resolve $\sim 4 \mathrm{eV}$ (and Athena half this value); using the bright $\mathrm{Fe} \mathrm{XXV}$ line at $6.7 \mathrm{keV}$, the lower detection limit becomes $\Delta E / E_{0} \sim 0.06$ percent (the dark regions in the right-hand panels of Fig. 4). Assuming good statistics, the maps show that Astro-H could detect turbulence in most of the cluster, where $\sigma_{1 \mathrm{D} \text {,ew }} \gtrsim 200 \mathrm{~km} \mathrm{~s}^{-1}$ or 1 D Mach number $\gtrsim 0.13$ (the non-black regions), covering our entire simulated range (see

\footnotetext{
5 The $\sqrt{2}$ term comes from the definition of the Gaussian distribution, $\propto \exp \left[-x^{2} / 2 \sigma^{2}\right]$, and not from isotropy arguments.
}

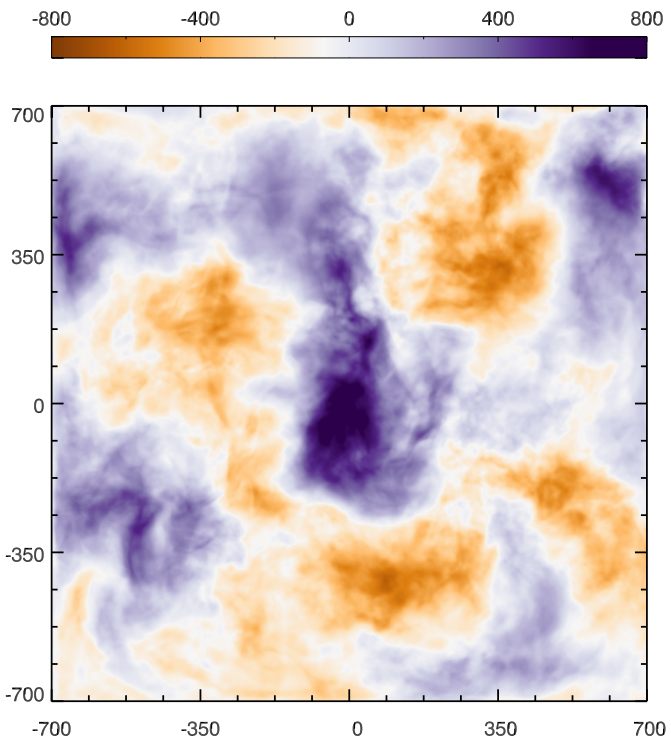

Fig. 6. X-ray emission-weighted velocity $\left(\mathrm{km} \mathrm{s}^{-1}\right)$ along the $y$-axis for the hydro model with $M \sim 0.5$ (compare with the first row in Fig. 4; the conductive models display similar maps.). The projected velocity highlights only the large-scale motions, which dominate the kinetic energy content in our (and cosmological) runs due to the injection at $L>100 \mathrm{kpc}$.

Nagai et al. 2013 for synthetic maps using Astro-H response). The Fe XXV is an excellent line, since the associated thermal broadening is just $\sigma_{\text {th }}=\left(k_{\mathrm{b}} T / 56 m_{\mathrm{p}}\right)^{0.5} \sim 120 \mathrm{~km} \mathrm{~s}^{-1}$; thereby, the turbulent dispersion typically dominates the contribution to the total broadening of this line.

While the projected velocity dispersion $\sigma_{1 \mathrm{D} \text {,ew }}$ highlights the small-scale motions via the line broadening, the projected X-ray emission-weighted velocity field probes the large-scale motions via the line shift (e.g. Zhuravleva et al. 2012). We notice that the driven velocity field still has an average 3D laminar motion $\sim 0$. Figure 6 shows the large eddies that are several $100 \mathrm{kpc}$, which carry most of the specific kinetic energy $\left(\sigma_{v}^{2} / 2 \sim A_{v, \max }^{2}\right)$ and would be absent in atmospheres stirred only at small scales. The relative strength of the line broadening and shift thus carries important information about the nature of the driven turbulence, which is a solid proxy corroborated by its insensitivity to conduction.

In passing, we note that X-ray observations are not the single channel to probe ICM fluctuations. We propose using the thermal Sunyaev-Zel'dovich (SZ) effect to independently extract pressure fluctuations. Current X-ray maps still have $\gtrsim 4 \times$ higher spatial resolution. However, future observations (e.g. ALMA, CCAT, SKA) will allow us to constrain the ICM power spectrum even in the submillimeter/radio band, avoiding the use of expensive X-ray spectroscopy.

\section{Discussion and physical interpretation}

In this section, we discuss the physical interpretation of the ICM power spectrum; in particular, we are concerned with the tight relation between the velocity and the other primary thermodynamic quantities (entropy, pressure, and density). It shall be kept in mind that the reason why we perform $3 \mathrm{D}$ simulations is that it is impossible to analytically solve a chaotic, nonlinear system. The following arguments arise from first order perturbations or dimensional theories and shall be regarded as simple 
estimates. The simulations generally confirm the ansatz presented below, albeit with relevant differences, which are critically discussed. We first focus on the normalization of the spectra $(l \sim L$; Sect. 5.1), and then we analyze the spectral cascade $(l<L$; Sect. 5.2), along with the alterations imparted by conduction from the ideal evolution.

\subsection{Spectra normalization}

The normalization of the spectra is likely related to the relative importance of gravity waves and sound waves. Linearizing the perturbed hydrodynamic equations, it is possible to describe the propagation of a general wave in a spherical and gravitationally stratified atmosphere in terms of the following dispersion relation (see Balbus \& Soker 1990, for the WKBJ analysis):

$\omega^{4}-\omega^{2} c_{\mathrm{s}}^{2} k^{2}+\omega_{\mathrm{BV}}^{2} c_{\mathrm{s}}^{2} k_{\perp}^{2}=0$,

where $k^{2}=k_{r}^{2}+k_{\perp}^{2}\left(k_{r}\right.$ and $k_{\perp}$ are the radial and azimuthal components of the wavenumber vector $\boldsymbol{k}$, respectively), $c_{\mathrm{s}}=$ $\left(\gamma k_{\mathrm{b}} T / \mu m_{\mathrm{p}}\right)^{1 / 2}$ is the adiabatic sound speed, and $\omega_{\mathrm{BV}}$ is the Brunt-Väisälä (buoyancy) frequency, defined as

$\omega_{\mathrm{BV}} \equiv\left[\frac{g}{\gamma} \frac{\mathrm{d} \ln K}{\mathrm{~d} r}\right]^{1 / 2}$,

where $g$ is the gravitational acceleration. Equation (2) includes the action of two key waves. The middle term is associated with pressure waves, or simply sound waves ( $p$-waves), while the last term represents gravity waves ( $g$-waves) driven by the restoring buoyant force. In the next sections, we show that small perturbations driven by the two waves are tied to the Mach number, $\sim \delta v / c_{\mathrm{s}}$. For both $g$ - and $p$-waves, this holds within order unity (as in the simulated nonlinear regime), but the leading perturbations and dynamical modes differ: $g$-waves mainly drive entropy perturbations $(\delta K / K)$, increase the gas vorticity, and induce a tangential bias in the turbulent velocity field; $p$-waves are instead associated with compressive pressure fluctuations $(\delta P / P)$, a preferentially irrotational field, and isotropic turbulence (or with slightly radial bias).

In Fig. 7, we show the frequency of the simulated turbulent motions, $t_{\text {turb }}^{-1}$, compared with the Brunt-Väisälä frequency (black; Eq. (3)) in the full radial range (at variance with GC13, where we focused on the properties of the central region). At large scales, the two frequencies tend to be roughly comparable (Froude number $\sim 1$ ); hence, both $g$-waves and $p$-waves can be excited. This is a typical condition for most clusters, since large-scale profiles (entropy and pressure) are fairly self-similar, and turbulence follows our simulated subsonic range. Let us first analyze the two limiting regimes to understand better both processes.

\subsubsection{Low frequencies (low $M)$ : $g$-waves}

The stratification of the ICM atmosphere allows the excitation of gravity waves (e.g. Lufkin et al. 1995; Ruszkowski \& Oh 2010, 2011). In the low frequency regime, the dispersion relation in Eq. (2) can be written as

$\omega^{2} \simeq \omega_{\mathrm{BV}}^{2} \frac{k_{\perp}^{2}}{k^{2}}$

which tells us that gravity waves are evanescent for $\omega>\omega_{\mathrm{BV}}$, since $k_{r}$ must be imaginary. Therefore, wherever $\omega<\omega_{\mathrm{BV}}$, $g$-waves are excited. For a cluster atmosphere, $\omega_{\mathrm{BV}}$ declines

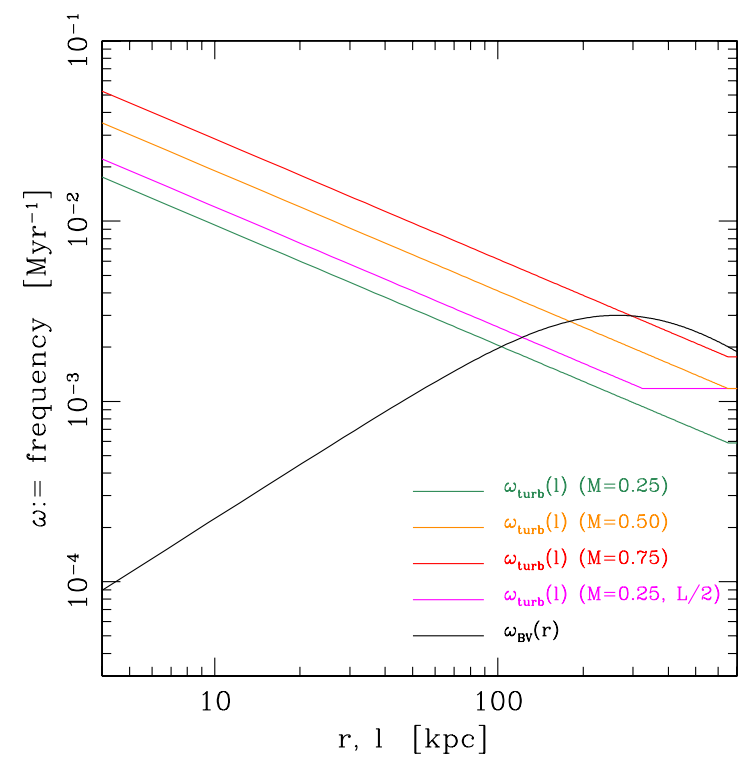

Fig. 7. Typical frequency of the turbulent motions including the cascade (for the simulated sample $M=0.25 \rightarrow 0.75$ ), compared with the Brunt-Väisälä buoyancy frequency (black). The minimum turbulence frequency is at the injection scale, typically $L \sim 600 \mathrm{kpc}$. For $\omega_{\text {turb }}<\omega_{\mathrm{BV}}$ (Froude $<1$ ), $g$-waves tend to dominate driving entropy fluctuations, while sound waves drive stronger pressure perturbations in the opposite regime. Both types of perturbations are $\propto M$.

at large $r$ and waves are trapped within the radius, such that $\omega \simeq \omega_{\mathrm{BV}}$ (Balbus \& Soker 1990) as $k \simeq k_{\perp}$. More importantly, buoyant oscillations damp the radial component of turbulence, inducing a tangential bias in the gas velocity field (Froude $<1$ ). In the limiting case, the chaotic motions should collapse in azimuthal shells (e.g. Ruszkowski \& Oh 2010). The profile of the velocity anisotropy parameter, $\beta \equiv 1-\sigma_{v_{\perp}}^{2} / 2 \sigma_{v_{r}}^{2}$ would show $\beta \ll 0$. Tangentially-biased vorticity is thus a good marker of the $g$-waves influence (see Sect. 5.1.3).

Gravity waves are mainly tied to entropy perturbations (see also Z14). Using $\omega_{\mathrm{BV}}$ as dominant frequency, the buoyant acceleration over a displacement $\delta r$ can be described with a simple harmonic oscillator:

$\ddot{r}_{\mathrm{b}}=-\omega_{\mathrm{BV}}^{2} \delta r=-\frac{c_{\mathrm{s}}^{2}}{\gamma^{2}\left|H_{P}\right| H_{K}} \delta r$

where $\omega_{\mathrm{BV}}$ is written ${ }^{6}$ as a function of both the scale height of entropy, $H_{K} \equiv \mathrm{d} r / \mathrm{d} \ln K$, and pressure, $H_{P} \equiv \mathrm{d} r / \mathrm{d} \ln P$. Physically, $g$-waves occur because an entropy element is displaced from its equilibrium position, $r_{0}$, thus inducing an opposite force that acts to restore the blob back to where the radial entropy is the same (clusters are convectively stable, $\nabla K>0$ ). The small displacement is thus linked to $\delta K / K \simeq(\mathrm{d} \ln K / \mathrm{d} r)_{0} \delta r$, i.e. $\delta r \simeq(\delta K / K) H_{K_{0}}$. The specific potential energy of the harmonic oscillator is $E_{\mathrm{b}}=\omega_{\mathrm{BV}}^{2}(\delta r)^{2} / 2$. Substituting $\omega_{\mathrm{BV}}$ and $\delta r$, we can write

$E_{\mathrm{b}} \simeq \frac{c_{\mathrm{s}}^{2}}{2 \gamma^{2}} \frac{H_{K_{0}}}{\left|H_{P_{0}}\right|}\left(\frac{\delta K}{K}\right)^{2}$.

6 Assuming hydrostatic equilibrium, which is valid for low $M, g=$ $-c_{\mathrm{s}}^{2} /\left(\gamma H_{P}\right)$; we notice that $H_{P}<0$ and $H_{K}>0$. 
Using $E_{\mathrm{b}}$ as an estimate for the average specific kinetic energy $\left(\bar{v}_{1 \mathrm{D}}^{2} / 2 \sim E_{\mathrm{b}}\right)$ finally yields

$\bar{M}_{1 \mathrm{D}} \sim \frac{1}{\gamma} \sqrt{\frac{H_{K_{0}}}{\left|H_{P_{0}}\right|}}\left|\frac{\delta K}{K}\right| \simeq O(1)\left|\frac{\delta K}{K}\right|$.

The last step arises because the ratio of the scale heights for an isothermal atmosphere is constant, $H_{K} /\left|H_{P}\right|=1 /(\gamma-1)=1.5$. In general, $H_{K} /\left|H_{P}\right|=\left|\alpha_{P}\right| / \alpha_{K} \simeq 1-2\left(\right.$ within $\left.r_{500}\right)$, where $\alpha_{P}$ and $\alpha_{K}$ are the slopes of the logarithmic radial profiles of pressure and entropy, respectively; $\alpha_{P}$ steepens with increasing radius (Arnaud et al. 2010), while $\alpha_{K} \simeq 1$.

Figure 8 shows the power spectra of all the thermodynamic quantities, including turbulent velocities, for the hydro and a conductive run in the low Mach regime. Considering the hydro model (top), the ratio of $M_{1 \mathrm{D}} \simeq M_{3 \mathrm{D}} / \sqrt{3}$ and $\delta K / K$ near the injection scale is 0.85 , in line with the estimate in Eq. (7). Evidently, only one component of velocity is acting as an efficient mixer, since cluster gradients are functions of $r$. Figure 8 clarifies that the low frequency regime corresponds to

$\left|\frac{\delta P}{P}\right| \ll\left|\frac{\delta K}{K}\right|$,

since slow motions tend to be in pressure equilibrium with the surroundings, which is helped by the convective stability of the ICM. The isobaric behavior also manifests in the relation between density and temperature, or entropy and density (both anticorrelated):

$\left|\frac{\delta \rho}{\rho}\right| \approx\left|\frac{\delta T}{T}\right|$ and $\left|\frac{\delta K}{K}\right| \approx \gamma\left|\frac{\delta \rho}{\rho}\right| \quad$ [isobaric].

Both relations are followed within $\lesssim 10$ percent, as shown in Fig. 8 (consistently with the Pearson analysis in GC13). Recall that if we halve the injection scale (Sect. 3), this slightly reduces the strength of $g$-waves as $L^{1 / 3}$, since $\omega_{\text {turb }} \propto L^{-1 / 3}$ approaches $\omega_{\mathrm{BV}}$ (Fig. 7). The ratio $A_{v} / A_{\rho}$ thus increases by the same factor (sound waves are still too weak to contribute).

In the presence of mild conduction, the normalization is unaltered for $f<10^{-2}$, although the intermediate cascade is damped by the increased diffusivity of the "tracer" (Sect. 5.2). For $f \gtrsim 10^{-2}$, the large-scale perturbations are also progressively driven toward the isothermal regime. Gravity waves can still induce a significant entropy contrast, but buoyancy is weakened by the increased diffusivity, tracing the shallower temperature gradient instead of $\boldsymbol{\nabla} K$ (Ruszkowski \& Oh 2010). The normalization of perturbations can thus decrease by a factor $\sim 2$. The relation between the different perturbations changes as

$\left|\frac{\delta \rho}{\rho}\right| \gg\left|\frac{\delta T}{T}\right|$ and $\left|\frac{\delta K}{K}\right| \approx(\gamma-1)\left|\frac{\delta \rho}{\rho}\right| \quad$ [isothermal].

Since turbulent regeneration is continuous and the ion-electron equilibration time is non-negligible, the pure isothermal regime is impossible to achieve. Nevertheless, as shown in Fig. 8 (bottom panel), the gap between entropy and density perturbations starts to shrink, as $T_{\mathrm{e}}$ fluctuations gradually lower (see also Fig. 9, bottom).

\subsubsection{High frequency (high $M)$ : $p$-waves}

In the opposite regime, at high frequency $(M>0.5)$, the dispersion relation (Eq. (2)) is shaped by the contribution of sound waves ( $p$-waves), which can be now written as

$\omega^{2} \simeq c_{\mathrm{s}}^{2} k^{2}$.
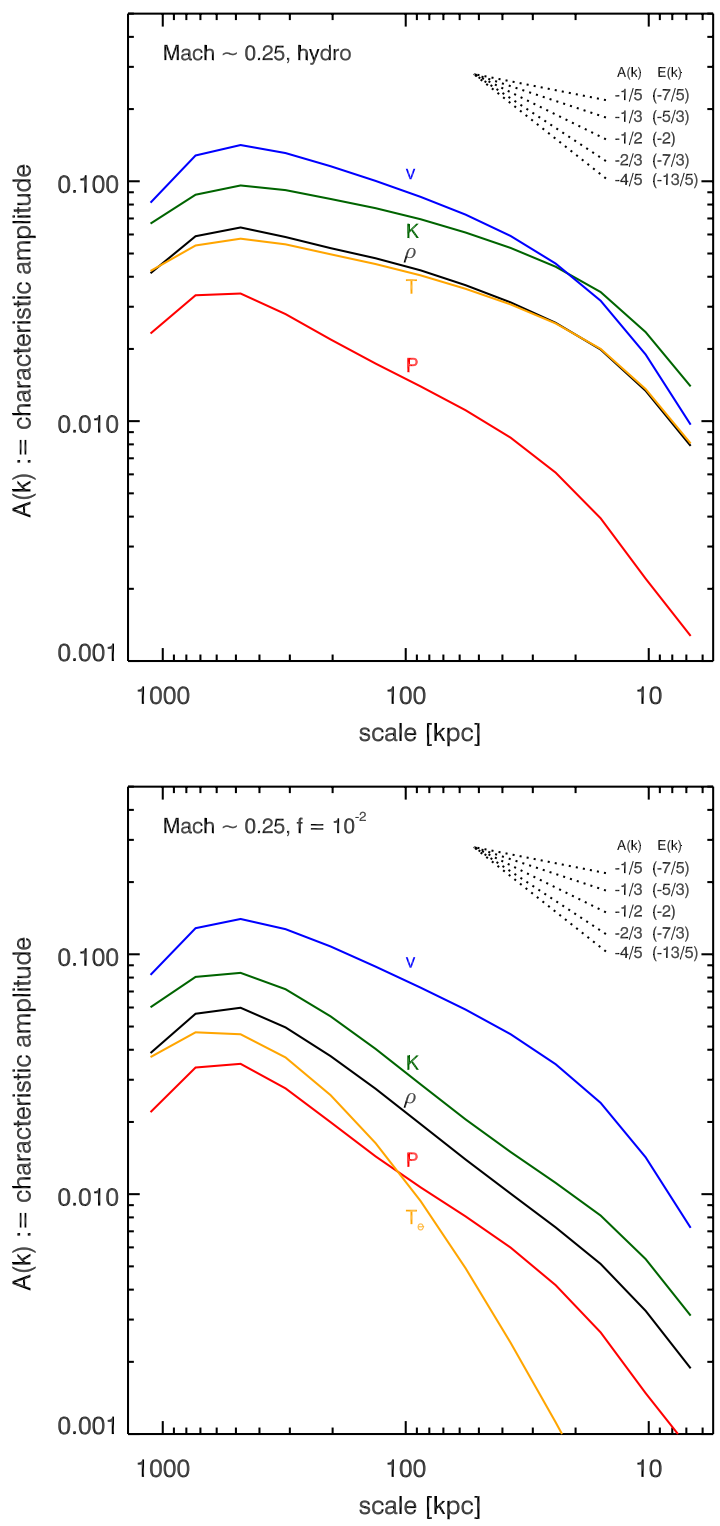

Fig. 8. Characteristic amplitude of the fluctuations related to all the thermodynamic quantities for the $M \sim 0.25$ flow without (top) and with conduction ( $f=10^{-2}$; bottom): $v / c_{\mathrm{s}}$ (3D Mach), $\delta K / K$ (entropy), $\delta \rho / \rho$ (density), $\delta T / T$ (temperature), $\delta P / P$ (pressure). Except for turbulent velocities (laminar motions are null by construction), all other quantities are divided by the azimuthally averaged profile. For the conductive runs, we plot the (observable) electron temperature; the entropy parameter is $K \equiv\left(P_{\mathrm{e}}+P_{\mathrm{i}}\right) / \rho^{\gamma}$. In the low $M$ flow, entropy perturbations (tied to $g$-waves) are the leading tracer of turbulent velocities, respecting the isobaric regime. Conduction gradually shifts the latter mode toward the isothermal regime, changing the relation with the derived thermodynamic quantities ( $\delta K / K$ starts to approach density fluctuations).

The azimuthal component of the wavenumber scales as $k_{\perp} \propto r^{-1}$. Therefore, $p$-waves excited in the cluster central regions become mainly radial further out, $k \sim k_{r}$. Moreover, disturbances to the vorticity results to be proportional to (Lufkin et al. 1995)

$\frac{\partial}{\partial t} \delta(\nabla \times \boldsymbol{v}) \propto \boldsymbol{k} \times \boldsymbol{\nabla} \ln K$, 
implying that $p$-waves are preferentially ${ }^{7}$ characterized by an irrotational velocity field, in contrast with the more tangential $g$-waves. Overall, stronger $p$-waves tend to restore isotropic turbulence, or to induce a slightly radial bias (depending on how many sound waves are excited in the central regions; Sect. 5.1.3).

The characteristic injection frequency of $p$-waves is $\omega_{s}^{2} \simeq$ $c_{\mathrm{s}}^{2} / L^{2}$. In this high frequency regime

$\left|\frac{\delta P}{P}\right|>\left|\frac{\delta K}{K}\right|$,

meaning that pressure perturbations drive the dynamics and fluctuations that follow the adiabatic regime (constant entropy). Following the same arguments provided in the previous section, the displacement magnitude is now tied to pressure variations as $\left|(\delta P / P) H_{P_{0}}\right|$. Using the potential energy as an estimate for the average kinetic energy (cf. Eqs. (6)-(7)) now yields

$\bar{M}_{1 \mathrm{D}} \sim \frac{\left|H_{P_{0}}\right|}{L}\left|\frac{\delta P}{P}\right| \simeq O(1)\left|\frac{\delta P}{P}\right|$,

where $\left|H_{P_{0}}\right| \simeq L /\left|\alpha_{P}\right|$, since we are analyzing the large-scale power. We note that stratification allows a more efficient generation of sound waves compared with uniform media (e.g. Stein 1967), as shown by the simulations, due to the partial conversion of solenoidal turbulence in more compressive motions.

Comparing the last estimate with Eq. (7), it is clear that the (1D) Mach number drives the spectrum normalization of perturbations in both cases, as found in the simulations. The transition must be smooth, as hinted by the general dispersion relation (Eq. (2)). However, the driving perturbations change character. Figure 9 shows that $\delta P / P$ rises linearly with $M$ for $M \gtrsim 0.5$, while $\delta K / K$ remains constant $\left(A_{K \text {, max }} \sim 0.15\right.$ in both hydro runs). In the $M \sim 0.75$ flow (middle panel), $\delta P / P$ has reached $\delta K / K$; hence, $p$-waves do not yet fully overcome $g$-waves, which would happen for $M \gtrsim 1$ (as $\omega_{\text {turb }}>\omega_{\mathrm{BV}}$ at each radius, or Froude $>1$; Fig. 7). Moreover, while gravity waves tend to accumulate in the system, $p$-waves may leave it in a few soundcrossing times. Again, turbulence with high Mach number is required to see a system fully dominated by $p$-waves.

According to Eq. (13), the perturbations of the other thermodynamic quantities start to shift from the isobaric to adiabatic regime, implying the following conversion:

$\left|\frac{\delta \rho}{\rho}\right| \approx \frac{1}{\gamma-1}\left|\frac{\delta T}{T}\right|$ and $\left|\frac{\delta P}{P}\right| \approx \gamma\left|\frac{\delta \rho}{\rho}\right| \quad$ [adiabatic],

as signaled by the increasing gap between density and temperature fluctuations (up to $\simeq 1.5$ ). Adding conduction (Fig. 9, bottom) shifts the adiabatic mode again toward a partial isothermal regime (Eq. (10)). Interestingly, pressure fluctuations are now more clearly the driver of density fluctuations $(\delta P / P \sim \delta \rho / \rho)$, since $\delta K / K$ has degraded by over $50 \%$ compared with the hydro run.

In the low $M$ regime, we note that the presence of weak $\delta P / P$ can be mainly attributed to the conservation of the Bernoulli parameter (although exactly valid only in the steady state). For a constant potential, it can be written as

$\frac{v^{2}}{2}+\frac{c_{\mathrm{s}}^{2}}{\gamma-1}=$ const.

Differentiating and taking $v_{0}=0$ as the reference velocity yields $\delta P / P \propto M^{2}$, which is indeed followed by the runs with $M<0.5$ (compare Figs. 8 and 9, top panels), while models with stronger turbulence follow $\delta P / P \propto M$ (Fig. 9, top and middle panels).

\footnotetext{
7 Vorticity can be generated in part via the baroclinic instability, as sound waves travel obliquely across the entropy gradient.
}
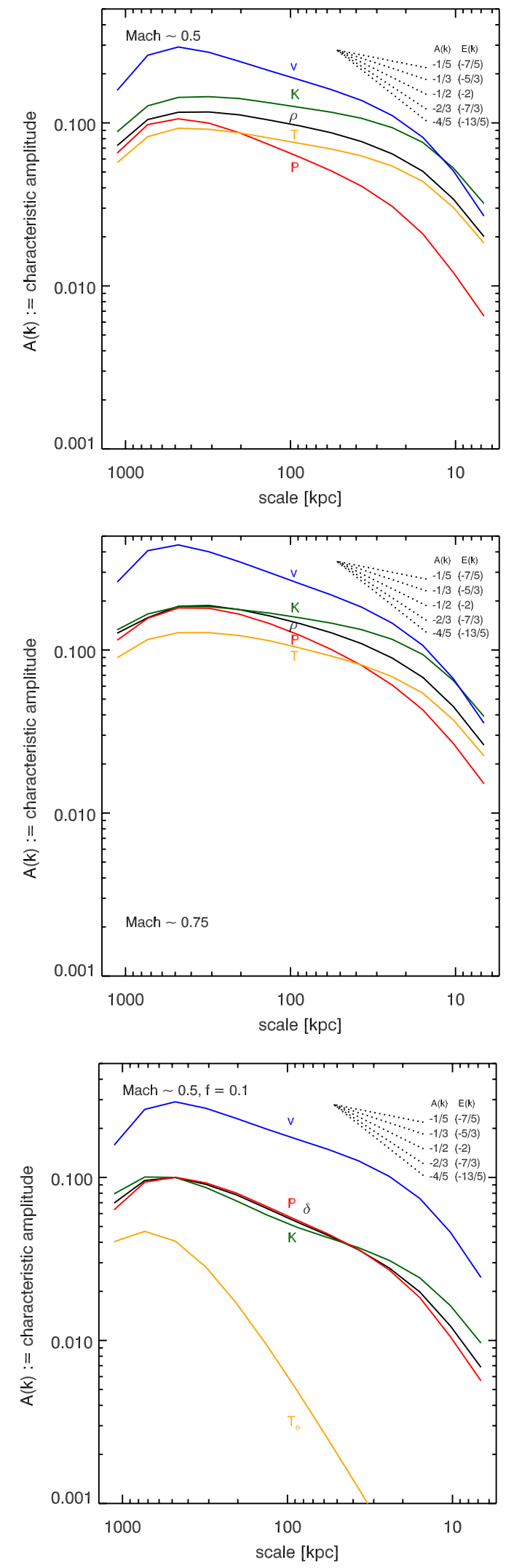

Fig. 9. Characteristic amplitude of all the thermodynamic fluctuations for the runs with $M \sim 0.5$ (hydro and $f=0.1$ ) and $M \sim 0.75$ (cf. Fig. 8). In significantly turbulent atmospheres, $p$-waves start to affect the fluctuations dynamics via $\delta P / P$, still in conjunction with entropy perturbations $\left(\omega_{\text {turb }}\right.$ is not yet $\left.\gg \omega_{\mathrm{BV}}\right)$. The derived quantities follow from the adiabatic/isothermal mode for the hydro/conductive flow. 


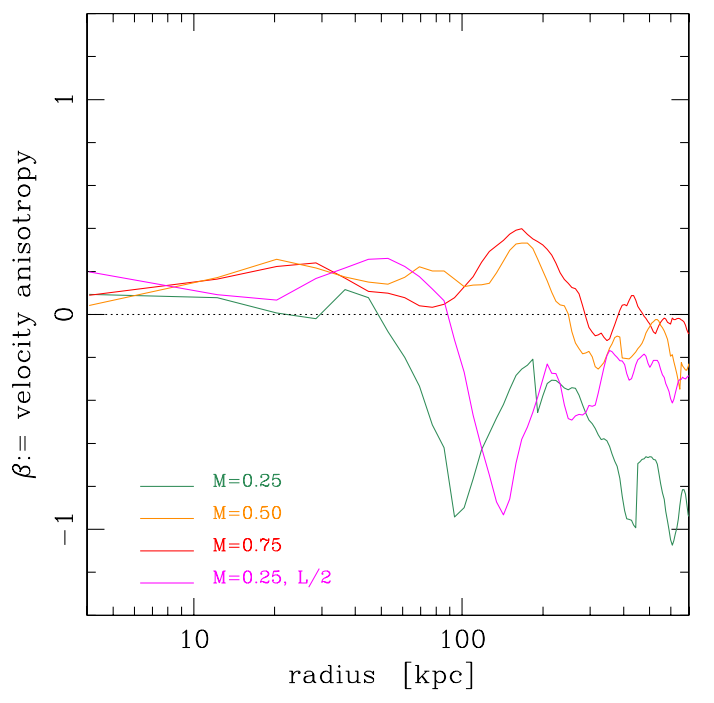

Fig. 10. Velocity anisotropy as a function of radius, $\beta(r) \equiv 1-\sigma_{v_{\perp}}^{2} / 2 \sigma_{v_{r}}^{2}$, for all the hydro models. Negative/positive values imply a tangential/radial bias. $g$-waves $(M \leq 0.25)$ tend to damp the radial component of turbulent motions, inducing a tangential bias; $p$-waves $(M \geq 0.75)$ tend instead to preserve isotropic motions, or to induce a slightly radial bias. In realistic clusters, the anisotropy is expected to be minor, due to the interplay of both waves and the recurrent stirring.

\subsubsection{Real systems: $g$ - and $p$-waves interplay}

The estimates in Eqs. (7) and (14) are crude approximations to reality. In the realistic cluster evolution, the 3D hydrodynamic equations and the related perturbations are nonlinear, with the addition of chaotic stirring (Sect. 5.2.1 for other deviations). Multiple frequencies act at the same time, describing waves at different scales and radii. Although the simulations confirm that the spectra normalization is provided by $M_{1 \mathrm{D}}$, we expect a combination of $g$-and $p$-waves that shape the dynamics of a turbulent cluster. This is visually highlighted by the maps in Fig. 4. More quantitatively, we can discriminate the action of both channels through an important marker, which is the anisotropy of velocities along the radial direction (Sects. 5.1.1-5.1.2). We notice that computing the velocity spectra over the scale $l$ obfuscates the radial anisotropy.

In Fig. 10, we show the $\beta \equiv 1-\sigma_{v_{\perp}}^{2} / 2 \sigma_{v_{r}}^{2}$ parameter for all the hydro models. For the low $M \sim 0.25$ flow, above $\sim 100 \mathrm{kpc}$, the motions are mildly ${ }^{8}$ tangential, reaching a minimum value $\beta \sim-1$. Analyzing the frequencies in Fig. 7, this corresponds to the region where $\omega<\omega_{\mathrm{BV}}$, that is where $g$-waves tend to be more dominant and damp the radial component of turbulent motions. Raising $M$ increases the turbulence frequency up to $\sim \omega_{\mathrm{BV}}$, even near the injection scale at several $100 \mathrm{~s} \mathrm{kpc}$. Therefore, the largescale motions progressively lose the tangential bias, drifting toward isotropy $(\beta \sim 0)$. In the $M \sim 0.75$ flow (red), $p$-waves start to dominate: motions show no sign of the tangential bias with instead a slightly radial bias (Sect. 5.1.2). Within $r<100 \mathrm{kpc}$, the frequencies of the turbulent cascade are always greater than $\omega_{\mathrm{BV}}$; hence, $p$-waves start to have a major influence. The transition occurs at smaller radii for lower $M$, as suggested by Fig. 10. On the other hand, large-scale $g$-waves tend to be trapped within the cluster core; the combination of the two effects results in a quasi isotropic $\beta$ in the core (or slightly radial $\beta \sim 0.2$ ). In the

\footnotetext{
8 The retrieved anisotropy is not strong, since $\beta \simeq-1$ corresponds to $v_{\phi} \simeq \sqrt{1-\beta} v_{r} \simeq 1.4 v_{r}$, which is difficult to observe by visual inspection.
}

presence of conduction, the same scenario applies, but the $\beta$ factor is overall reduced toward the isotropic value (by about half the previous values). As noted in Sect. 5.1.1, thermal conduction indeed inhibits buoyancy.

Overall, we suggest using the $\beta$ parameter to discriminate the effects of $g$-and $p$-waves, in conjunction with the thermodynamic mode. We remark that typical cluster conditions are expected to show at best mild anisotropic motions, which emerge only in spherical coordinates; most clusters are not in a regime in which $\omega \ll \omega_{\mathrm{BV}}$ (or the opposite), where radial motions are dramatically suppressed. The recurrent stirring also promotes $\beta$ values drifting toward isotropy. Nevertheless, the ICM spectrum normalization is always comparable to $M_{1 \mathrm{D}}$ within order unity, regardless of which one is the driving wave, for both the linear approximation (Eq. (2)) and the nonlinear simulations.

\subsection{Spectral cascade: advection of tracers}

We have analyzed the physical interpretation of the spectra normalization $(l \sim L)$ so far. The next question is why perturbations show an inertial cascade similar to that of velocities $(l<L)$. The main thermodynamic variables can be crudely considered as "tracers" of the velocity field, whose spectra are explained with the advection theory of passive scalars (e.g. Sreenivasan 1991; Monin \& Yaglom 1975; Warhaft 2000, for a review). This is particularly relevant in the subsonic regime, since the compressive term $\boldsymbol{\nabla} \cdot \boldsymbol{v} \rightarrow 0$.

The equation governing the advection of a passive incompressible scalar $C$ is given by (Warhaft 2000, Sect. 1)

$\frac{D C}{D t} \equiv \frac{\partial C}{\partial t}+\boldsymbol{v} \cdot \nabla C=\boldsymbol{\nabla} \cdot(\kappa \nabla C)$,

where $\kappa$ is the diffusivity of the tracer and $D / D t$ is the Lagrangian derivative. According to classic KolmogorovObukhov-Corrsin theory (KOC; Obukhov 1949; Corrsin 1951; Warhaft 2000), the energy spectrum of the scalar linearly traces that of velocities, such as

$E_{C}(k) \propto E_{v}(k) \propto k^{-5 / 3}$.

Considering a 2D vortical motion, the vorticity is conserved (see also Kelvin's theorem), $D(\boldsymbol{\nabla} \times \boldsymbol{v}) / D t=0$, in analogy to Eq. (17). The turbulent eddies and the scalar are thus expected to share similar properties, like the spectral cascade, although the diffusive term introduces some discrepancies (Sect. 5.2.1).

Entropy is an excellent example of "passive tracer". The quantity $S \equiv k_{\mathrm{B}} /\left[(\gamma-1) \mu m_{\mathrm{p}}\right] \ln K$ has indeed the advantage of being insensitive to adiabatic compressions or expansions. Aside from diffusion, the Lagrangian derivative of entropy is thus conserved, if no irreversible heating $(\mathcal{H})$ or cooling $(\mathcal{L})$ occurs, such as

$\rho T \frac{\mathrm{D} S}{\mathrm{D} t} \equiv \rho T\left(\frac{\partial S}{\partial t}+\boldsymbol{v} \cdot \nabla S\right)=\mathcal{H}-\mathcal{L} \simeq \boldsymbol{\nabla} \cdot\left(D_{\text {turb }} \rho T \nabla S\right)$.

The hot ICM has negligible radiative cooling $(\mathcal{L} \simeq 0$ ); the only source of heating can be turbulent diffusion or dissipation. The latter is subdominant for subsonic flows, $t_{\text {diss, heat }} \simeq M^{-2} t_{\text {turb }}$. The entropy $S$ can thus replace the scalar $C$ in Eq. (17) with a diffusivity tied to the turbulent field, $D_{\text {turb }} \sim \sigma_{v} l$ (Sect. 2). In the low $M$ regime (Sect. 5.1.1), entropy fluctuations tend to lead the dynamics of perturbations, which are linked to the largescale $g$-waves. The $\delta K / K$ cascade then develops over $l<L$, tracing the velocity inertial regime (Fig. 8) in line with KOC 
theory (Eq. (18)). Since the fluctuations of density and temperature follow from the dominant mode - isobaric (Eq. (9)), isothermal (Eq. (10)), or adiabatic (Eq. (15)) - also their cascade traces that of $\delta K / K$. As discussed before, increasing the Mach number boosts the impact of $p$-waves. The leading tracer gradually shifts toward $\delta P / P$ fluctuations. In the $M \sim 0.75$ hydro run (Fig. 9), density perturbations start to track more closely the $\delta P / P$ cascade. Pressure is affected by adiabatic processes, yet the simulations show that its cascade is slightly steeper than that of velocities, signaling that it can be used as a crude tracer of the (subsonic) flow. Interestingly, pressure is not directly affected by turbulent mixing (which acts on entropy) and, thus, displays a tighter cascade with velocities ${ }^{9}$.

Adding conduction, increases the effective diffusivity of the tracer $\left(\kappa \rightarrow f \kappa_{\mathrm{S}}\right)$, leading to a cascade steeper than that of Kolmogorov (Fig. 8, bottom; notice how $T_{\mathrm{e}}$ fluctuations are strongly damped). The decline of the tracer spectrum starts to occur as $D_{\text {cond }}>D_{\text {turb }} / 100$, i.e. $P_{\mathrm{t}}<100$. Physically, the quick increase in temperature induces a rapid re-expansion of the forming overdensity, which overcomes the action of turbulent regeneration. In the conductive regime, the $A_{v} / A_{\rho}$ ratio is thus expected to gradually increase as a function of $f$ (Fig. 2). The ratio widens up to a factor of 3-5 over all scales in the presence of strong conduction $(f \gtrsim 0.1)$.

It is interesting to note that in the simple KOC picture:

$E(k)_{C}=b \bar{\epsilon}_{C}^{-1 / 3} \bar{\epsilon}_{v} k^{-5 / 3}$,

where $b$ is a universal constant. The average dissipation rate of the passive tracer and velocity is $\bar{\epsilon}_{C} \sim \sigma_{C}^{2} /\left(L / \sigma_{v}\right)$ and $\bar{\epsilon}_{v} \sim \sigma_{v}^{3} / L$, respectively, implying that the normalization of Eq. (20) is independent of $\sigma_{v}$. In reality, the compressive term sustains a relation between the density variance and the Mach number, even in homogeneous media: in the subsonic range, $\delta \rho / \rho$ fade as $M^{2}$ (cf. Sect. 5.1.2), while the relation becomes linear for supersonic turbulence (see Kowal et al. 2007 and Sect. 1). The latter is, however, conceptually different from our retrieved linear relation developing in the subsonic state of galaxy clusters. In the stratified ICM plasma, increasing the Mach number implies larger coherent displacement, leading to a larger contrast of entropy/pressure defined by the cluster gradients or scale heights (Eqs. (7) and (14)).

\subsubsection{KOC departures and the radial gradients}

Although KOC theory can explain the overall picture of the spectral cascade, its arguments are purely based on dimensional analysis. Our retrieved slope of entropy/density is typically shallower than the Kolmogorov index (in the non-diffusive models). Physical experiments (e.g. Fig. 5 in Sreenivasan 1991) show that the tracer slope approaches the Kolmogorov cascade only for very high Reynolds numbers and in a slow asymptotic way. For low Reynolds numbers, as in our ICM simulations $\left(R_{L} \lesssim 500\right.$; GC13, Sect. 2.6), the spectral index is expected to be shallow (cf. Fig. 4 in Warhaft 2000). Even pressure fluctuations, albeit in line with the Kolmogorov cascade, are shallower than the classic expectation $E_{P} \propto k^{-7 / 3}\left(A_{P} \propto k^{-2 / 3}\right.$; Schuecker et al. 2004), in the $M \gtrsim 0.5$ runs. A slope as shallow as $A_{k} \propto k^{-1 / 5}$ signals that the timescale for transferring the tracer variance from large to small scales is $\propto k^{-4 / 5}$, instead of the Kolmogorov $\propto k^{-2 / 3}$, due to diffusion effects affecting the transfer process in different ways

\footnotetext{
9 In the $M \sim 0.25$ run, the $\delta P / P$ cascade is steeper, following the classical $E_{P} \propto k^{-7 / 3}$, which is solely driven by the Bernoulli term (Eq. (16)).
}

(see the viscosity test in Fig. 3). This is remarked by the uncorrelated phases between velocity and the tracer (Fig. 4).

Another departure from the KOC cascade may be associated to compressive features. In the extreme case of highly supersonic turbulence, shocks induce very thin peaks in gas density (Kim \& Ryu 2005, Fig. 2). Sharp peaks can be seen as delta functions, which generate a flat spectrum, $P_{\delta} \sim k^{0}$, in Fourier space. The ICM turbulence is, however, subsonic; thus, the contribution of thin compressive features to our observed flattening is limited.

The incomplete similarity with the Kolmogorov cascade more likely depends on the initial entropy/pressure gradients. Eqs. (7) and (14) are only valid for small displacements. For a nonlinear evolution, the injected eddy experiences $H_{K}$ and $H_{P}$ which varies with radius, given that the initial cluster profiles are self-similar power laws (Sect. 5.1.1):

$\frac{\delta K}{K}=\alpha_{K} \frac{\delta r}{r}$ and $\frac{\delta P}{P}=\alpha_{P} \frac{\delta r}{r}$.

For instance, fixing $\delta r \sim L$, the injected turbulence at smaller radii can create relatively larger contrasts, inducing a flattening in the spectral cascade. The magnitude of this effect depends, however, on the relation $r \longleftrightarrow l$. Since chaotic motions are 3D, the turbulent streamlines intersect different projections of the radial gradients, hence the dependence shall be weak. Neglecting the previous KOC departures, the flattening of the simulated spectra corresponds to an average $r \sim l^{0.13}$. An opposing effect is related to the fact that the indices $\alpha_{K}$ and $\alpha_{P}$ are not constant, but both decline within the core radius (turbulent mixing also slightly lowers $\alpha_{K}$ in time; GC13). According to Eq. (21), a lower slope implies a lower contrast. This may explain why the $A_{v} / A_{\rho}$ ratio slowly declines toward smaller scales (Fig. 2, top), though always remaining larger than unity. We will investigate other cluster atmospheres in the future to assess the impact of different $K$ and $P$ scale heights. In closing, we note that all these secondary effects are washed out in the presence of any significant diffusivity, which completely inverts the $A_{v} / A_{\rho}$ downtrend (Fig. 2).

\subsection{Further improvements}

Finally, we discuss the limitations of the models and further improvements. We studied the evolution of the ICM, primarily in the hot regime. In future works, we plan to extend the simulated sample (as strong cool-core systems) and to test additional physics. Needless to say, 3D high-resolution 2T simulations with turbulence and diffusive terms are extremely expensive, hence small steps must be taken.

It will be interesting to include the effect of cooling, which can induce thermal instability and condensation of cold filaments (Gaspari et al. 2012a, 2013). The AGN feedback balances cooling, preserving the cluster core in global quasi-thermal equilibrium (e.g. Gaspari et al. 2012b). However, both processes just affect the inner region $r<0.1 R_{500}$ (cf. Gaspari et al. 2014), while galaxy clusters maintain self-similarity at large radii, especially in the entropy profile ( $\alpha_{K} \sim 1$; e.g. Panagoulia et al. 2014). We thus do not expect dramatic deviations from the current ICM power spectrum (which is intrinsically volume-weighted), and we believe our results can be applied to a wide range of clusters and conditions. Strongly unrelaxed systems, such as as major mergers, might present significant variations, due to the dynamic gravitational potential for example, and requires to be further tested. We are also studying the role of very small injection scales (e.g. AGN outflows): for $L<50 \mathrm{kpc}$, entropy perturbations may be considerably weaker (as $\omega_{\mathrm{BV}}<\omega_{\text {turb }}$ ), while 
pressure perturbations should drive the normalization of the density power spectrum, even at low Mach numbers.

In the companion work (Z14), we improve the driving, including the cosmological evolution and the turbulence generated by mergers and large-scale inflows. Albeit limited by low resolution, we find that the linear $M_{1 \mathrm{D}}-\delta \rho / \rho$ relation holds across a large sample of simulated clusters. We retrieve a relation scatter of $\sim 30$ percent. In the cosmological context, it is more difficult to disentangle the source of the velocity anisotropy, especially in unrelaxed systems. As for observational data, it is important to accurately remove the underlying radial profile and the strongly nonlinear sub-structures, which can contaminate the large-scale power. We find that the most reliable scales dominated by the turbulent cascade are $l<300 \mathrm{kpc}$, which is fortunately the optimal regime for X-ray observations (see GC13, Sect. 4.3 for a comparison with real data).

We plan to test additional physics. We currently probed the effects of (nearly maximal) Spitzer-like viscosity and electronion equilibration. It will be interesting to assess the role of the related magnetic suppression factors (which can be different from that of heat transport). On the other hand, diffusivities linked to the ions are roughly two orders of magnitude slower compared with electron thermal conduction, since the electron sound speed is $\simeq 43$ times that of ions. We thus expect conduction to dominate the shape of the power spectrum over a large range of scales. A viscosity lower than the present Spitzer-like value (which would require a much higher resolution) would imply a more extended inertial cascade. Compared with Fig. $2, A_{v} / A_{\rho}$ should thus differ only below $10 \mathrm{~s} \mathrm{kpc}$, continuing to widen in combination with high conductivity. In the presence of both low viscosity and conductivity, density and velocity spectra are expected to be tightly coupled again (KOC theory).

Fully MHD simulations are a further route of improvement, which can better model the local features (as cold fronts and filaments). However, besides the numerical complication of integrating anisotropic conduction for long times, the MHD runs can at best retrieve the geometric suppression factor (see Sect. 2). Therefore, we would still be forced to parametrize the conductivity with a factor $f_{\|}$to include microinstabilities and line divergence below the gas mean free path.

\section{Conclusions}

We carried out 3D high-resolution hydrodynamic 2T simulations to study the power spectrum of the hot ICM in its various manifestations. We focused on the properties of the velocity field and the intimate relation with the driven thermodynamic fluctuations (in particular of density, the primary observable). The ICM power spectrum contains enough information to accurately constrain the dominant physics of the diffuse medium, as the strength of turbulent motions, the level of thermal diffusivity, and the thermodynamic mode, among the most notable. The spectra of $v / c_{\mathrm{s}}$ and of perturbations (e.g. $\delta \rho / \rho$ ) are overall self-similar, varying the strength of turbulence via the 3D Mach number, $M$, or changing the injection scale, $L$.

At the large cluster scales $(l \sim L)$, several $100 \mathrm{kpc}$, we retrieve the following:

- Weak turbulent motions in the cluster $(M \lesssim 0.25)$ mainly excite gravity waves $\left(\omega_{\mathrm{turb}}<\omega_{\mathrm{BV}}\right)$; the leading perturbations are related to entropy variations $\delta K / K$. For stronger turbulence $(M>0.5)$, sound waves start to significantly contribute ( $\left.\omega \gtrsim \omega_{\mathrm{BV}}\right)$, passing the leading role to the compressive pressure fluctuations $\delta P / P$.
- The other thermodynamic perturbations, such as $\delta \rho / \rho$ and $\delta T / T$, derive from the dominant mode of the process: isobaric (for $g$-waves/low $M$ ), adiabatic (for $p$-waves/high $M$ ), or a mixed state for intermediate $M$. Conduction shifts the perturbations toward the isothermal mode.

- In both the regimes driven by $g$ - or $p$-waves, the turbulent 1D Mach number is comparable to the variance of the leading perturbations $(K$ or $P$ ) within order unity. For example, $M_{1 \mathrm{D}} \sim \delta K / K \sim \gamma \delta \rho / \rho$ for $M \lesssim 0.25$ flows. Quantitatively, all simulations show a linear relation given by $A_{v, \text { max }} \simeq 2.3 A_{\rho \text {, max }}\left(\right.$ at $L \sim 600 \mathrm{kpc}$ ) with a weak $L^{1 / 3}$ scaling. To convert between Fourier and real space, the relation to apply is instead $M \approx 4 A_{\rho, \max }$ (at $L \sim 600 \mathrm{kpc}$ ).

- Turbulent motions with a tangential bias $(\beta(r)<0)$ mark the influence of $g$-waves (low $M$ ), while $p$-waves (high $M$ ) tend to preserve isotropy or to induce a slightly radial bias. Most clusters show intermediate Mach numbers, hence we expect a mixed regime drifting toward global isotropy (Froude $\sim 1$ ).

At the intermediate/small scales $(l<L), 10-100 \mathrm{kpc}$, we find the following:

- The turbulent velocities develop a Kolmogorov cascade $\left(A_{v} \propto k^{-1 / 3}\right.$ or $\left.E_{v} \propto k^{-5 / 3}\right)$ in all subsonic runs, despite stratification $\left(\omega_{\mathrm{turb}}<\omega_{\mathrm{BV}}\right)$. The thermodynamic perturbations, particularly entropy, act as effective "tracers" of the velocity field, developing an analogous inertial cascade, in line with the classic (Kolmogorov-Obukhov-Corrsin) advection theory of passive scalars in turbulent media.

- The cluster radial gradients, in conjunction with compressive features, conspire to moderately flatten the perturbations spectrum, slightly departing from the KOC theory and inducing a slow decrease in $A_{v} / A_{\rho}$.

- Thermal conduction strongly damps density/entropy perturbations (the spectral steepening occurs where Prandtl $P_{\mathrm{t}}<$ $100)$ but leaves the velocity cascade unaltered. This has a dramatic consequence on $A_{v} / A_{\rho}$, which inverts the downtrend shown in the non-diffusive model. The ratio can widen up to $\sim 5$, as a function of $P_{\mathrm{t}}^{-1} \propto f / M$, unveiling the presence of significant conductivity in the ICM and breaking any degeneracy in the interpretation of single spectra.

The real-space and projected maps carry important information:

- The ideal or poorly diffusive flows $\left(f \lesssim 10^{-2}\right)$ show complex filamentary and patchy density/entropy structures (similar in the core and outskirts), which are excited by the large-scale waves and later altered by hydrodynamical instabilities. The conductive models, however, show smooth maps due to the smearing of sharp features, which does not affect the turbulent eddies. Albeit sharing similar amplitude, velocities have uncorrelated phases with the tracer, hence a filament does not necessarily imply a high local velocity.

- The thermodynamic fluctuations can be described by lognormal distributions with weak non-Gaussian deviations, strengthening the role of the power spectrum.

- Synthetic X-ray images of velocity dispersion show that the forthcoming Astro-H (and Athena) will be able to well detect subsonic ICM turbulence. Using the broadening of the Fe XXV line, the detectable turbulent broadening is $\gtrsim 200 \mathrm{~km} \mathrm{~s}^{-1}\left(M_{1 \mathrm{D}} \gtrsim 0.13\right.$ for massive clusters), which can probe density perturbations on the order of a few percent and allows a precise calibration of the linear relation. The projected velocity maps (linked to the line shift) highlight the power stored in the large-scale motions, constraining the injection scale. 
The analysis presented in this work shows the wealth of information that can be extracted from the ICM power spectrum. In Coma cluster, Schuecker et al. (2004) retrieve pressure fluctuations, which are mildly adiabatic and trace a Kolmogorov spectrum, in line with a $M \sim 0.4$ turbulent flow. In Gaspari \& Churazov (2013, see also Churazov et al. 2012), we showed that the density spectrum arising from deep Chandra data of Coma is consistent with a similar level of turbulence (several $100 \mathrm{~s} \mathrm{~km} \mathrm{~s}^{-1}$ ), along with highly suppressed conduction $\left(f \sim 10^{-3}\right)$. Sanders \& Fabian (2012) found density fluctuations ( $₫ 8$ percent) having a cascade shallower than Kolmogorov in the AWM7 cluster, which implies highly suppressed conduction and $M \lesssim 0.18$. Being able to quickly convert between thermodynamic properties and gas motions through a simple linear relation, or being able to assess the plasma diffusivity through the spectral slope or the $A_{v} / A_{\rho}$ diagnostic, is a powerful tool for both observational and theoretical study. The same analysis can be extended to other gaseous halos, such as massive galaxies and groups. Although current constraints are in its embryonic stage, we are beginning to understand the richness of information that the ICM power spectrum can convey. Future studies and observations (not only in the X-ray band but also via SZ maps) will be able to improve and exploit the full potential of the ICM power spectrum, helping us to probe the physics of the gaseous medium with high precision.

Acknowledgements. The FLASH code was in part developed by the DOE NNSA-ASC OASCR Flash center at the University of Chicago. M.G. is grateful for the financial support provided by the Max Planck Fellowship. We acknowledge the MPA, RZG, and CLS center for the availability of high-performance computing resources. D.N. and E.L. acknowledge support from NSF grant AST1009811, NASA ATP grant NNX11AE07G, NASA Chandra grants GO213004B and TM4-15007X, and the Research Corporation. M.G. thanks A. Schekochihin, R. Sunyaev, S. Borgani, F. Brighenti, F. Miniati, D. Eckert, S. Molendi, X. Shi, E. Pointecouteau for helpful comments, and the anonymous referee for a highly positive feedback.

\section{References}

Arévalo, P., Churazov, E., Zhuravleva, I., Hernández-Monteagudo, C., \& Revnivtsev, M. 2012, MNRAS, 426, 1793

Armstrong, J. W., Cordes, J. M., \& Rickett, B. J. 1981, Nature, 291, 561

Armstrong, J. W., Rickett, B. J., \& Spangler, S. R. 1995, ApJ, 443, 209

Arnaud, M., Pratt, G. W., Piffaretti, R., et al. 2010, A\&A, 517, A92

Balbus, S. A., \& Soker, N. 1990, ApJ, 357, 353

Borgani, S., \& Kravtsov, A. 2011, Adv. Sci. Lett., 4, 204

Brunetti, G., \& Lazarian, A. 2007, MNRAS, 378, 245

Chandran, B. D. G., \& Cowley, S. C. 1998, Phys. Rev. Lett., 80, 3077

Churazov, E., Forman, W., Jones, C., Sunyaev, R., \& Böhringer, H. 2004, MNRAS, 347, 29

Churazov, E., Forman, W., Vikhlinin, A., et al. 2008, MNRAS, 388, 1062 Churazov, E., Vikhlinin, A., Zhuravleva, I., et al. 2012, MNRAS, 421, 1123 Corrsin, S. 1951, J. Appl. Phys., 22, 469

de Plaa, J., Zhuravleva, I., Werner, N., et al. 2012, A\&A, 539, A34

Dennis, T. J., \& Chandran, B. D. G. 2005, ApJ, 622, 205

Dolag, K., Jubelgas, M., Springel, V., Borgani, S., \& Rasia, E. 2004, ApJ, 606, L97

Dolag, K., Vazza, F., Brunetti, G., \& Tormen, G. 2005, MNRAS, 364, 753

Ettori, S., \& Fabian, A. C. 2000, MNRAS, 317, L57

Federrath, C., Banerjee, R., Clark, P. C., \& Klessen, R. S. 2010, ApJ, 713, 269

Forman, W., Jones, C., Churazov, E., et al. 2007, ApJ, 665, 1057

Fryxell, B., Olson, K., Ricker, P., et al. 2000, ApJS, 131, 273

Gaspari, M., \& Churazov, E. 2013, A\&A, 559, A78

Gaspari, M., Melioli, C., Brighenti, F., \& D'Ercole, A. 2011a, MNRAS, 411, 349
Gaspari, M., Brighenti, F., D'Ercole, A., \& Melioli, C. 2011b, MNRAS, 415, 1549

Gaspari, M., Ruszkowski, M., \& Sharma, P. 2012a, ApJ, 746, 94

Gaspari, M., Brighenti, F., \& Temi, P. 2012b, MNRAS, 424, 190

Gaspari, M., Ruszkowski, M., \& Oh, S. P. 2013, MNRAS, 432, 3401

Gaspari, M., Brighenti, F., Temi, P., \& Ettori, S. 2014, ApJ, 783, L10

Inogamov, N. A., \& Sunyaev, R. A. 2003, Astron. Lett., 29, 791

Kawahara, H., Suto, Y., Kitayama, T., et al. 2007, ApJ, 659, 257

Kim, D.-W., \& Fabbiano, G. 2003, ApJ, 586, 826

Kim, J., \& Ryu, D. 2005, ApJ, 630, L45

Kim, K.-T., Kronberg, P. P., Dewdney, P. E., \& Landecker, T. L. 1990, ApJ, 355, 29

Komarov, S. V., Churazov, E. M., Schekochihin, A. A., \& ZuHone, J. A. 2014 MNRAS, 440, 1153

Konstandin, L., Girichidis, P., Federrath, C., \& Klessen, R. S. 2012, ApJ, 761, 149

Kowal, G., Lazarian, A., \& Beresnyak, A. 2007, ApJ, 658, 423

Lau, E. T., Kravtsov, A. V., \& Nagai, D. 2009, ApJ, 705, 1129

Lufkin, E. A., Balbus, S. A., \& Hawley, J. F. 1995, ApJ, 446, 529

Markevitch, M., \& Vikhlinin, A. 2007, Phys. Rep., 443, 1

Marsch, E., \& Tu, C.-Y. 1990, J. Geophys. Res., 95, 11945

McDonald, M., \& Veilleux, S. 2009, ApJ, 703, L172

Miniati, F. 2014, ApJ, 782, 21

Monin, A. S., \& Yaglom, A. M. 1975, Statistical fluid mechanics: Mechanics of turbulence - Vol. 2 (Cambridge, Mass.: MIT Press)

Nagai, D., Vikhlinin, A., \& Kravtsov, A. V. 2007, ApJ, 655, 98

Nagai, D., Lau, E. T., Avestruz, C., Nelson, K., \& Rudd, D. H. 2013, ApJ, 777, 137

Nandra, K., Barret, D., Barcons, X., et al. 2013 [arXiv: 1306.2307]

Narayan, R., \& Medvedev, M. V. 2001, ApJ, 562, L129

Norman, M. L., \& Bryan, G. L. 1999, in The Radio Galaxy Messier 87, eds. H.-J. Röser, \& K. Meisenheimer (Berlin: Springer Verlag), Lect. Notes Phys., 530, 106

Obukhov, A. M. 1949, Zv. Akad. Nauk. SSSR, Ser. Geogr. Geophys., 13, 58

Padoan, P., Jones, B. J. T., \& Nordlund, A. P. 1997, ApJ, 474, 730

Panagoulia, E. K., Fabian, A. C., \& Sanders, J. S. 2014, MNRAS, 438, 2341

Planck Collaboration XVI. 2014, A\&A, in press, DOI: 10.1051/0004-6361/201321591

Rebusco, P., Churazov, E., Böhringer, H., \& Forman, W. 2005, MNRAS, 359, 1041

Rechester, A. B., \& Rosenbluth, M. N. 1978, Phys. Rev. Lett., 40, 38

Roediger, E., Kraft, R. P., Nulsen, P., et al. 2013, MNRAS, 436, 1721

Ruszkowski, M., \& Oh, S. P. 2010, ApJ, 713, 1332

Ruszkowski, M., \& Oh, S. P. 2011, MNRAS, 414, 1493

Sanders, J. S., \& Fabian, A. C. 2012, MNRAS, 421, 726

Sanders, J. S., \& Fabian, A. C. 2013, MNRAS, 429, 2727

Scannapieco, E., \& Brüggen, M. 2008, ApJ, 686, 927

Schmidt, W., Almgren, A. S., Braun, H., et al. 2014, MNRAS, 440, 3051

Schuecker, P., Finoguenov, A., Miniati, F., Böhringer, H., \& Briel, U. G. 2004, A\&A, 426, 387

Shi, X., \& Komatsu, E. 2014, MNRAS, 442, 521

Smith, B., O'Shea, B. W., Voit, G. M., Ventimiglia, D., \& Skillman, S. W. 2013, ApJ, 778, 152

Spitzer, L. 1962, Physics of Fully Ionized Gases (New York: Interscience Wiley) Sreenivasan, K. R. 1991, Roy. Soc. London Proc. Ser. A, 434, 165

Stein, R. F. 1967, Sol. Phys., 2, 385

Takahashi, T., Mitsuda, K., Kelley, R., et al. 2010, in SPIE Conf. Ser., 7732

Tamura, T., Yamasaki, N. Y., Iizuka, R., et al. 2014, ApJ, 782, 38

Valdarnini, R. 2011, A\&A, 526, A158

Vazza, F., Brunetti, G., Kritsuk, A., et al. 2009, A\&A, 504, 33

Vazza, F., Brunetti, G., Gheller, C., Brunino, R., \& Brüggen, M. 2011, A\&A, 529, A17

Vikhlinin, A., Kravtsov, A., Forman, W., et al. 2006, ApJ, 640, 691

Voigt, L. M., \& Fabian, A. C. 2004, MNRAS, 347, 1130

Warhaft, Z. 2000, Ann. Rev. Fluid Mech., 32, 203

Woo, R., \& Armstrong, J. W. 1979, J. Geophys. Res., 84, 7288

Zakamska, N. L., \& Narayan, R. 2003, ApJ, 582, 162

Zhuravleva, I., Churazov, E., Kravtsov, A., \& Sunyaev, R. 2012, MNRAS, 422, 2712

Zhuravleva, I., Churazov, E., Kravtsov, A., et al. 2013, MNRAS, 428, 3274

Zhuravleva, I., Churazov, E. M., Schekochihin, A. A., et al. 2014, ApJ, 788, L13

ZuHone, J. A., Markevitch, M., Ruszkowski, M., \& Lee, D. 2013, ApJ, 762, 69 